\title{
The mean width of random polytopes circumscribed around a convex body
}

\author{
Károly J. Böröczky, Ferenc Fodor and Daniel Hug
}

\begin{abstract}
Let $K$ be a $d$-dimensional convex body and let $K^{(n)}$ be the intersection of $n$ halfspaces containing $K$ whose bounding hyperplanes are independent and identically distributed. Under suitable distributional assumptions, we prove an asymptotic formula for the expectation of the difference of the mean widths of $K^{(n)}$ and $K$, and another asymptotic formula for the expectation of the number of facets of $K^{(n)}$. These results are achieved by establishing an asymptotic result on weighted volume approximation of $K$ and by 'dualizing' it using polarity.
\end{abstract}

\section{Introduction}

Let $K$ be a convex body (compact convex set with nonempty interior) in $d$-dimensional Euclidean space $\mathbb{R}^{d}$. The convex hull $K_{(n)}$ of $n$ independent random points in $K$ chosen according to the uniform distribution is a common model of a random polytope contained in $K$. The famous four-point problem of Sylvester [43] is the starting point of an extensive investigation of random polytopes of this type. Beside specific probabilities as in Sylvester's problem, important objects of study are expectations, variances and distributions of various geometric functionals associated with $K_{(n)}$. Typical examples of such functionals are volume, other intrinsic volumes, and the number of $i$-dimensional faces. In their ground-breaking papers $[\mathbf{3 1}, \mathbf{3 2}]$, Rényi and Sulanke considered random polytopes in the Euclidean plane and proved asymptotic results for the expectations of basic functionals of random polytopes in a convex domain $K$ in the cases where $K$ is sufficiently smooth or a convex polygon. Since then most results have been in the form of asymptotic formulae as the number $n$ of random points tends to infinity.

In the last three decades, much effort has been devoted to exploring the properties of this particular model of a random polytope contained in a $d$-dimensional convex body $K$. For instance, for a sufficiently smooth convex body $K$, asymptotic formulae were proved for the expectation of the mean width difference $W(K)-W\left(K_{(n)}\right)$ by Schneider and Wieacker [38], and for the volume difference $V(K)-V\left(K_{(n)}\right)$ by Bárány [1]. The assumption of smoothness was relaxed in the case of the mean width by Böröczky, Fodor, Reitzner and Vígh [10], and removed by Schütt [40] in the case of the volume. General intrinsic volumes are treated in [11] under a weak smoothness assumption. Recently, even variance estimates, laws of large numbers and central limit theorems have been proved in different settings in a sequence of contributions, for instance by Bárány, Reitzner, Schreiber, Vu and Yukich; see $[\mathbf{3}, \mathbf{5}, \mathbf{2 9}, \mathbf{3 0}$, 39, 44, 45]. For more details on the current state-of-the-art of this line of research, see the

Received 23 September 2008; revised 13 October 2009.

2000 Mathematics Subject Classification 52A22 (primary), 60D05, 52A27 (secondary).

The first author was supported by OTKA grants 068398 and 075016 , by the EU Marie Curie projects BudAlgGeo (MTKD-CT-2004-002988) and DiscConvGeo (MTKD-CT-2005-014333), and by the János Bolyai Research Scholarship of the Hungarian Academy of Sciences. The second author was supported by the János Bolyai Research Scholarship of the Hungarian Academy of Sciences and by OTKA grants 068398 and 075016. The third author was supported by the European Network PHD, FP6 Marie Curie Actions, RTN, Contract MCRN-CT-2004-511953. 
survey papers by Weil and Wieacker [46], Gruber [16] and Schneider [36], and the recent monograph of Schneider and Weil [37].

In a third paper, Rényi and Sulanke [33] suggested a model that is 'dual' to the model of a random polytope contained in a given convex body $K$ (a random inscribed polytope), that is, they considered a random polytope containing a given convex body (a random circumscribed polytope). Subsequently, this approach has not received as much attention as the inscribed case', although it is closely related to linear optimization (cf. $[\mathbf{6}, \mathbf{3 4}, \S 6])$. There are various ways of producing circumscribed random polytopes containing a given convex body. In this paper, we consider a model in which the circumscribed polytope arises as an intersection of closed halfspaces whose bounding hyperplanes are randomly chosen hyperplanes. A precise description of this probability model is given in Section 2, and a more general setting is considered in Section 5. Here we just provide the following rough description. In Euclidean space $\mathbb{R}^{d}$, we consider hyperplanes that intersect the parallel domain at radius one of a given convex body $K$ but miss the interior of $K$, and we use the corresponding restriction of the (suitably normalized) Haar measure on the set of hyperplanes in $\mathbb{R}^{d}$ to provide an associated probability measure. For $n$ independent random hyperplanes $H_{1}, \ldots, H_{n}$, chosen according to this distribution, the intersection of the closed halfspaces bounded by $H_{1}, \ldots, H_{n}$ and containing $K$ determines a circumscribed random polyhedral set containing $K$ (which might be unbounded). The main goal of this paper is to find asymptotic formulae for the expectation of the difference of the mean widths of a random circumscribed polytope and the given convex body $K$, and for the expectation of the number of facets of a circumscribed random polyhedral set. These (and more general) results will be achieved by establishing general results on weighted volume approximation of a given convex body by inscribed random polytopes. In all these results, no regularity or curvature assumptions on $K$ are required.

As for earlier results, we mention the paper by Ziezold [50], who investigated circumscribed polygons in the plane, and the doctoral dissertation of Kaltenbach [22], who proved asymptotic formulae for the expectation of the volume difference and for the expectation of the number of vertices of circumscribed random polytopes around a convex body, under the assumption that the boundary of the reference body $K$ is sufficiently smooth. Recently, Böröczky and Schneider [9] established upper and lower bounds for the expectation of the mean width difference for a general convex body $K$. Furthermore, they also proved asymptotic formulae for the expected number of vertices and facets of $K^{(n)}$, and an asymptotic formula for the expectation of the mean width difference, under the assumption that the reference body $K$ is a simplicial polytope with $r$ facets (cf. [2] for a related contribution).

In [8], Böröczky and Reitzner discuss a different model of a random circumscribed polytope where $n$ independent random points are chosen from the boundary of a given smooth convex body $K$, and the intersection of the supporting halfspaces of $K$ at these points is the random polyhedral set under consideration. This framework is again dual to the one considered by Schütt and Werner (see [42]), who study the expected volume of the convex hull of $n$ independent random points chosen from the boundary of a convex body satisfying a weak regularity assumption.

\section{The probability space and the main goal}

We first describe the setting for stating our results on circumscribed random polyhedral sets. Throughout this paper, $K$ denotes a compact convex set with interior points (a convex body) in $d$-dimensional Euclidean space $\mathbb{R}^{d}(d \geqslant 2)$. We write $\langle\cdot, \cdot\rangle$ for the scalar product and $\|\cdot\|$ for the norm in $\mathbb{R}^{d}$. For background on convexity, we refer to the monographs by Schneider [35] or by Gruber [17]. Let $V$ denote volume and let $\mathcal{H}^{j}$ denote the $j$-dimensional Hausdorff measure. The unit ball of $\mathbb{R}^{d}$ with center at the origin $o$ is denoted by $B^{d}$, and $S^{d-1}$ is its boundary. 
We consider $\alpha_{d}:=V\left(B^{d}\right)$ and $\omega_{d}:=\mathcal{H}^{d-1}\left(S^{d-1}\right)=d \alpha_{d}$. The parallel body of $K$ of radius 1 is $K_{1}:=K+B^{d}$. Let $\mathcal{H}$ denote the space (with its usual topology) of hyperplanes in $\mathbb{R}^{d}$ and let $\mathcal{H}_{K}$ be the subspace of hyperplanes meeting $K_{1}$ but not the interior of $K$. For $H \in \mathcal{H}_{K}$, the closed halfspace bounded by $H$ that contains $K$ is denoted by $H^{-}$. Let $\mu$ denote the motion invariant Borel measure on $\mathcal{H}$, normalized so that $\mu(\{H \in \mathcal{H}: H \cap M \neq \emptyset\})$ is the mean width $W(M)$ of $M$, for every convex body $M \subset \mathbb{R}^{d}$. Let $2 \mu_{K}$ be the restriction of $\mu$ to $\mathcal{H}_{K}$. Since $\mu\left(\mathcal{H}_{K}\right)=W\left(K+B^{d}\right)-W(K)=W\left(B^{d}\right)=2$, the measure $\mu_{K}$ is a probability measure. For $n \in \mathbb{N}$, let $H_{1}, \ldots, H_{n}$ be independent random hyperplanes in $\mathbb{R}^{d}$, that is, independent $\mathcal{H}$-valued random variables on some probability space $(\Omega, \mathbf{A}, \mathbb{P})$, each with distribution $\mu_{K}$. The possibly unbounded intersection

$$
K^{(n)}:=\bigcap_{i=1}^{n} H_{i}^{-}
$$

of the halfspaces $H_{i}^{-}$, with $H_{i} \in \mathcal{H}_{K}$ for $i=1, \ldots, n$, is a random polyhedral set. A major aim of the present work is to investigate $\mathbb{E} W\left(K^{(n)} \cap K_{1}\right)$, where $\mathbb{E}$ denotes mathematical expectation. The intersection with $K_{1}$ is considered, since $K^{(n)}$ is unbounded with positive probability. Instead of $\mathbb{E} W\left(K^{(n)} \cap K_{1}\right)$, we could consider $\mathbb{E}_{1} W\left(K^{(n)}\right)$, the conditional expectation of $W\left(K^{(n)}\right)$ under the condition that $K^{(n)} \subset K_{1}$. Since $\mathbb{E} W\left(K^{(n)} \cap K_{1}\right)=\mathbb{E}_{1} W\left(K^{(n)}\right)+O\left(\gamma^{n}\right)$ with $\gamma \in(0,1)$ (cf. [9]), there is no difference in the asymptotic behaviors of both quantities, as $n \rightarrow \infty$. We also remark that, for the asymptotic results, the parallel body $K_{1}$ could be replaced by any other convex body containing $K$ in its interior; this would only affect some normalization constants.

Let $\partial K$ denote the boundary of $K$. We call $\partial K$ twice differentiable in the generalized sense at a boundary point $x \in \partial K$, if there exists a quadratic form $Q$ on $\mathbb{R}^{d-1}$, the second fundamental form of $K$ at $x$, with the following property. If $K$ is positioned in such a way that $x=o$ and $\mathbb{R}^{d-1}$ is a support hyperplane of $K$ at $o$, then in a neighborhood of $o$ we see that $\partial K$ is the graph of a convex function $f$ defined on a $(d-1)$-dimensional ball around $o$ in $\mathbb{R}^{d-1}$ satisfying

$$
f(z)=\frac{1}{2} Q(z)+o\left(\|z\|^{2}\right),
$$

as $z \rightarrow o$. Alternatively, we call $x$ a normal boundary point of $K$. If this is the case, we write $\kappa(x)=\operatorname{det}(Q)$ to denote the generalized Gaussian curvature of $K$ at $x$. Writing $\kappa(x)$, we always assume that $\partial K$ is twice differentiable in the generalized sense at $x \in \partial K$. According to a classical result of Alexandrov (see $[\mathbf{1 7}, \mathbf{3 5}]), \partial K$ is twice differentiable in the generalized sense almost everywhere with respect to the boundary measure of $K$ (in other words, $\mathcal{H}^{d-1}$-almost all boundary points are normal boundary points). Finally, we define the constant

$$
c_{d}=\frac{\left(d^{2}+d+2\right)\left(d^{2}+1\right)}{2(d+3) \cdot(d+1) !} \Gamma\left(\frac{d^{2}+1}{d+1}\right)\left(\frac{d+1}{\alpha_{d-1}}\right)^{2 /(d+1)}
$$

(cf. [49]), which will appear in the statements of our main results. In the following, we simply write $d x$ instead of $\mathcal{H}^{d}(d x)$.

The main asymptotic result concerning the expected difference of the mean widths of $K^{(n)}$ and $K$ is the following theorem. Generalizations of Theorem 2.2, and also of Theorem 2.1 below, which hold under more general distributional assumptions, are provided in Section 5 . There we also indicate the connection to the $p$-affine surface area of a convex body.

Theorem 2.1. If $K$ is a convex body in $\mathbb{R}^{d}$, then

$$
\lim _{n \rightarrow \infty} n^{2 /(d+1)} \mathbb{E}\left(W\left(K^{(n)} \cap K_{1}\right)-W(K)\right)=2 c_{d} \omega_{d}^{-(d-1) /(d+1)} \int_{\partial K} \kappa(x)^{d /(d+1)} \mathcal{H}^{d-1}(d x) .
$$


Let $f_{i}(P)$, where $i \in\{0, \ldots, d-1\}$, denote the number of $i$-dimensional faces of a polyhedral set $P$. In the statement of the following theorem, $K^{(n)}$ could be replaced by the intersection of $K^{(n)}$ with a fixed polytope containing $K$ in its interior without changing the right-hand side. Alternatively, instead of $\mathbb{E}\left(f_{d-1}\left(K^{(n)}\right)\right)$ we could consider the conditional expectation of $f_{d-1}\left(K^{(n)}\right)$ under the assumption that $K^{(n)}$ is contained in $K_{1}$.

Theorem 2.2. If $K$ is a convex body in $\mathbb{R}^{d}$, then

$$
\lim _{n \rightarrow \infty} n^{-(d-1) /(d+1)} \mathbb{E}\left(f_{d-1}\left(K^{(n)}\right)\right)=c_{d} \omega_{d}^{-(d-1) /(d+1)} \int_{\partial K} \kappa(x)^{d /(d+1)} \mathcal{H}^{d-1}(d x) .
$$

Both theorems will be deduced from a 'dual' result on weighted volume approximation of convex bodies by inscribed random polytopes. This result is stated in the subsequent section. The usefulness of duality in random or best approximation has previously been observed for example in $[\mathbf{1 2}, \mathbf{1 5}, \mathbf{2 2}]$.

\section{Weighted volume approximation by inscribed polytopes}

For a given convex body, we introduce a class of inscribed random polytopes. Let $C$ be a convex body in $\mathbb{R}^{d}$, let $\varrho$ be a bounded nonnegative measurable function on $C$ and let $\mathcal{H}^{d}\llcorner C$ denote the restriction of $\mathcal{H}^{d}$ to $C$. Assuming that $\int_{C} \varrho(x) \mathcal{H}^{d}(d x)>0$, we choose random points from $C$ according to the probability measure given by

$$
\mathbb{P}_{\varrho, C}:=\left(\int_{C} \varrho(x) d x\right)^{-1} \varrho \mathcal{H}^{d}\llcorner C .
$$

Expectation with respect to $\mathbb{P}_{\varrho, C}$ is denoted by $\mathbb{E}_{\varrho, C}$. The convex hull of $n$ independent and identically distributed random points with distribution $\mathbb{P}_{\varrho, C}$ is denoted by $C_{(n)}$ if $\varrho$ is clear from the context. This yields a general model of an inscribed random polytope.

Generalizing a result by Schütt [40], we prove the following theorem.

Theorem 3.1. For a convex body $K$ in $\mathbb{R}^{d}$, a probability density function $\varrho$ on $K$, and an integrable function $\lambda: K \rightarrow \mathbb{R}$ such that, on a neighborhood of $\partial K$ relative to $K, \lambda$ and $\varrho$ are continuous and $\varrho$ is positive, we have

$$
\lim _{n \rightarrow \infty} n^{2 /(d+1)} \mathbb{E}_{\varrho, K} \int_{K \backslash K_{(n)}} \lambda(x) d x=c_{d} \int_{\partial K} \varrho(x)^{-2 /(d+1)} \lambda(x) \kappa(x)^{1 /(d+1)} \mathcal{H}^{d-1}(d x),
$$

where $c_{d}$ is defined in $(2.2)$.

The limit on the right-hand side of (3.1) depends only on the values of $\varrho$ and $\lambda$ on the boundary of $K$. In particular, we may prescribe any continuous positive function $\varrho$ on $\partial K$. Then any continuous extension of $\varrho$ to a probability density on $K$ (there always exists such an extension) will satisfy Theorem 3.1 with the prescribed values of $\varrho$ on the right-hand side.

Our proof of Theorem 3.1 is inspired by the approach in $[40]$, where the special case $\varrho \equiv \lambda \equiv 1$ is considered. We note that for [40, Lemma 2], which is crucial for the proof in [40], no explicit proof is provided, but reference is given to an analogous result in an unpublished note by $\mathrm{M}$. Schmuckenschläger. Besides a missing factor $\frac{1}{2}$, Lemma 2 does not hold in the generality stated in [40]. For instance, it is not true for simplices. Most probably, this gap can be overcome, but still our approach to prove Theorem 3.1, where [40, Lemma 2] is replaced by the present more elementary Lemma 4.2, might be of some interest. 
The present partially new approach to Theorem 3.1 involves also some other interesting new features. In particular, we do not need the concept of a Macbeath region. An outline of the proof is given below. It should also be emphasized that the generality of Theorem 3.1 is needed for our study of circumscribed random polyhedral sets via duality.

A classical argument going back to Efron [13] shows that

$$
\mathbb{E}_{\varrho, K}\left(f_{0}\left(K_{(n)}\right)\right)=n \cdot \mathbb{E}_{\varrho, K} \int_{K \backslash K_{(n-1)}} \varrho(x) d x,
$$

which yields the following consequence of Theorem 3.1.

Corollary 3.2. For a convex body $K$ in $\mathbb{R}^{d}$, and for a probability density function $\varrho$ on $K$ which is continuous and positive on a neighborhood of $\partial K$ relative to $K$, we have

$$
\lim _{n \rightarrow \infty} n^{-(d-1) /(d+1)} \mathbb{E}_{\varrho, K}\left(f_{0}\left(K_{(n)}\right)\right)=c_{d} \int_{\partial K} \varrho(x)^{(d-1) /(d+1)} \kappa(x)^{1 /(d+1)} \mathcal{H}^{d-1}(d x),
$$

where $c_{d}$ is defined in $(2.2)$.

The proof of Theorem 3.1 is obtained through the following intermediate steps. Details are provided in Section 4. Since the convex body $K$ is fixed, we write $\mathbb{E}_{\varrho}$ and $\mathbb{P}_{\varrho}$ instead of $\mathbb{E}_{\varrho, K}$ and $\mathbb{P}_{\varrho, K}$, respectively. The basic observation to prove Theorem 3.1 is that

$$
\mathbb{E}_{\varrho} \int_{K \backslash K_{(n)}} \lambda(x) d x=\int_{K} \mathbb{P}_{\varrho}\left(x \notin K_{(n)}\right) \lambda(x) d x,
$$

which is an immediate consequence of Fubini's theorem. Throughout the proof, we may assume that $o \in \operatorname{int}(K)$. The asymptotic behavior, as $n \rightarrow \infty$, of the right-hand side of (3.2) is determined by points $x \in K$ which are sufficiently close to the boundary of $K$. In order to give this statement a precise meaning, scaled copies of $K$ are introduced as follows. For $t \in(0,1)$, we define $K_{t}:=(1-t) K$ and $y_{t}:=(1-t) y$ for $y \in \partial K$. In Lemma 4.3, we show that

$$
\lim _{n \rightarrow \infty} n^{2 /(d+1)} \int_{K_{n^{-1 /(d+1)}}} \mathbb{P}_{\varrho}\left(x \notin K_{(n)}\right) \lambda(x) d x=0 .
$$

This limit relation is based on a geometric estimate of $\mathbb{P}_{\varrho}\left(x \notin K_{(n)}\right)$, provided in Lemma 4.1, and on a disintegration result stated as Lemma 4.2.

For $y \in \partial K$, we write $u(y)$ for some exterior unit normal of $K$ at $y$. This exterior unit normal is uniquely determined for $\mathcal{H}^{d-1}$-almost all boundary points of $K$. Applying the disintegration result again and using Lebesgue's dominated convergence result, we finally get

$$
\lim _{n \rightarrow \infty} n^{2 /(d+1)} \mathbb{E}_{\varrho} \int_{K \backslash K_{(n)}} \lambda(x) d x=\int_{\partial K} \lambda(y) J_{\varrho}(y) \mathcal{H}^{d-1}(d y),
$$

where

$$
J_{\varrho}(y)=\lim _{n \rightarrow \infty} \int_{0}^{n^{-1 /(d+1)}} n^{2 /(d+1)}\langle y, u(y)\rangle \mathbb{P}_{\varrho}\left(y_{t} \notin K_{(n)}\right) d t
$$

for $\mathcal{H}^{d-1}$-almost all $y \in \partial K$. For the subsequent analysis, it is sufficient to consider a small cap of $K$ at a normal boundary point $y \in \partial K$. The case $\kappa(y)=0$ is treated in Lemma 4.4. The main case is $\kappa(y)>0$. Here we reparametrize $y_{t}$ as $\tilde{y}_{s}$, in terms of the probability content of a small cap of $K$ whose bounding hyperplane passes through $y_{t}$. This implies that

$$
\begin{aligned}
J_{\varrho}(y)= & (d+1)^{-(d-1) /(d+1)} \alpha_{d-1}^{-2 /(d+1)} \varrho(y)^{-2 /(d+1)} \kappa(y)^{1 /(d+1)} \\
& \times \lim _{n \rightarrow \infty} \int_{0}^{n^{-1 / 2}} n^{2 /(d+1)} \mathbb{P}_{\varrho}\left(\tilde{y}_{s} \notin K_{(n)}\right) s^{-(d-1) /(d+1)} d s,
\end{aligned}
$$


cf. (4.26). It is then a crucial step in the proof to show that the remaining integral asymptotically is independent of the particular convex body $K$, and thus the limit of the integral is the same as for a Euclidean ball (see Lemma 4.6). To achieve this, the integral is first approximated, up to a prescribed error of order $\varepsilon>0$, by replacing $\mathbb{P}_{\varrho}\left(\tilde{y}_{s} \notin K_{(n)}\right)$ by the probability of an event that depends only on a small cap of $K$ at $y$ and on a small number of random points. This important step is accomplished in Lemma 4.5. For the proofs of Lemmas 4.5 and 4.6 it is essential that the boundary of $K$ near the normal boundary point $y$ can be suitably approximated by the osculating paraboloid of $K$ at $y$.

\section{Proof of Theorem 3.1}

To start with the actual proof, we fix some further notation. For $y \in \partial K$ and $t \in(0,1)$, we define the cap $C(y, t):=\left\{x \in K:\langle u(y), x\rangle \geqslant\left\langle u(y), y_{t}\right\rangle\right\}$ whose bounding hyperplane passes through $y_{t}$ and has normal $u(y)$. For $u \in \mathbb{R}^{d} \backslash\{o\}$ and $t \in \mathbb{R}$, we define the hyperplane $H(u, t):=\{x \in$ $\left.\mathbb{R}^{d}:\langle x, u\rangle=t\right\}$, and the closed halfspaces $H^{+}(u, t):=\left\{x \in \mathbb{R}^{d}:\langle x, u\rangle \geqslant t\right\}$ and $H^{-}(u, t):=$ $\left\{x \in \mathbb{R}^{d}:\langle x, u\rangle \leqslant t\right\}$ bounded by $H(u, t)$. We denote by $h(K, \cdot)=h_{K}$ the support function of $K$, that is, we have $h(K, u)=\max \{\langle x, u\rangle: x \in K\}$ for $u \in \mathbb{R}^{d}$.

For $y \in \partial K$, we denote by $r(y)$ the maximal number $r \geqslant 0$ such that $y-r u(y)+r B^{d} \subset$ $K$. This number is called the interior reach of the boundary point $y$. It is well known that $r(y)>0$ for $\mathcal{H}^{d-1}$-almost all $y \in \partial K$. If $r(y)>0$, then there is a unique tangent plane of $K$ at $y$. In particular, $r(y) \leqslant r(K)$, where $r(K)$ is the inradius of $K$. The convex hull of subsets $X_{1}, \ldots, X_{r} \subset \mathbb{R}^{d}$ and points $z_{1}, \ldots, z_{s} \in \mathbb{R}^{d}$ is denoted by $\left[X_{1}, \ldots, X_{r}, z_{1}, \ldots, z_{s}\right]$.

For real functions $f$ and $g$ defined on the same space $I$, we write $f \ll g$ or $f=O(g)$ if there exists a positive constant $\gamma$, depending only on $K, \varrho$ and $\lambda$, such that $|f| \leqslant \gamma \cdot g$ on $I$. In general, we write $\gamma_{0}, \gamma_{1}, \ldots$ to denote positive constants depending only on $K, \varrho$ and $\lambda$. The Landau symbol $o(\cdot)$ is defined as usual. We further consider $\mathbb{R}^{+}:=[0, \infty)$.

Finally, we observe that there exists a constant $\gamma_{0} \in(0,1)$ such that, for $y \in \partial K$, we have

$$
|\langle y, u(y)\rangle| \geqslant \gamma_{0}\|y\| \text {, and hence }\left\|y \mid u(y)^{\perp}\right\| \leqslant \sqrt{1-\gamma_{0}^{2}} \cdot\|y\|,
$$

where $y \mid u^{\perp}$ denotes the orthogonal projection of $y$ onto the orthogonal complement of the vector $u \in \mathbb{R}^{d} \backslash\{o\}$. Subsequently, we always assume that $n \in \mathbb{N}$.

Lemma 4.1. There exists a constant $\delta>0$, depending on $K$ and $\varrho$, such that if $y \in \partial K$ and $t \in(0, \delta)$, then

$$
\mathbb{P}_{\varrho}\left(y_{t} \notin K_{(n)}\right) \ll\left(1-\gamma_{1} r(y)^{(d-1) / 2} t^{(d+1) / 2}\right)^{n} .
$$

Remarks. (i) In addition, we may assume that on $K \backslash \operatorname{int}\left(K_{\delta}\right)$, both functions $\varrho$ and $\lambda$ are continuous, $\varrho$ is positive and $\gamma_{1} r(K)^{(d-1) / 2} \delta^{(d+1) / 2}<1$. Further, we always choose $\delta<1$.

(ii) In the following, we use the notion of a 'coordinate corner'. Given an orthonormal basis in a linear $i$-dimensional subspace $L$, the corresponding $(i-1)$-dimensional coordinate planes cut $L$ into $2^{i}$ convex cones, which we call coordinate corners (with respect to $L$ and the given basis).

Proof of Lemma 4.1. If $r(y)=0$, then there is nothing to prove. Therefore let $r(y)>$ 0 , thence $u(y)$ is uniquely determined. Choose an orthonormal basis in $u(y)^{\perp}$, and let $\Theta_{1}^{\prime}, \ldots, \Theta_{2^{d-1}}^{\prime}$ be the corresponding coordinate corners in $u(y)^{\perp}$. For $i=1, \ldots, 2^{d-1}$ and $t \in[0,1]$, we define

$$
\Theta_{i, t}:=C(y, t) \cap\left(y_{t}+\left[\Theta_{i}^{\prime}, \mathbb{R}^{+} y\right]\right)
$$


If $\delta>0$ is small enough to ensure that $\varrho>0$ is positive and continuous in a neighborhood (relative to $K$ ) of $\partial K$, then

$$
\int_{\Theta_{i, t}} \varrho(x) d x \geqslant \gamma_{2} V\left(\Theta_{i, t}\right)
$$

If $y_{t} \notin K_{(n)}$ and $o \in K_{(n)}$, then there exists a hyperplane $H$ through $y_{t}$, bounding the halfspaces $H^{-}$and $H^{+}$, for which $K_{(n)} \subset H^{-}$. Moreover, there is some $i \in\left\{1, \ldots, 2^{d-1}\right\}$ such that $\Theta_{i, t} \subset$ $H^{+}$. Therefore

$$
\mathbb{P}_{\varrho}\left(y_{t} \notin K_{(n)}, o \in K_{(n)}\right) \ll \sum_{i=1}^{2^{d-1}}\left(1-\gamma_{2} V\left(\Theta_{i, t}\right)\right)^{n} .
$$

Finally, we prove that

$$
V\left(\Theta_{i, t}\right) \gg r(y)^{(d-1) / 2} t^{(d+1) / 2},
$$

for $i=1, \ldots, 2^{d-1}$. According to (4.1), there exist positive constants $\gamma_{3}$ and $\gamma_{4}$ with $\gamma_{3} \leqslant 1$ such that if $t \leqslant \gamma_{3} r(y)$, then $\left(y_{t}+\Theta_{i}^{\prime}\right) \cap K$ contains a $(d-1)$-ball of radius at least

$$
\gamma_{4} \sqrt{r(y)^{2}-(r(y)-t)^{2}} \geqslant \gamma_{4} \sqrt{r(y) t},
$$

and we are done. On the other hand, if $t \geqslant \gamma_{3} r(y)$, then

$$
V\left(\Theta_{i, t}\right) \gg t^{d} \gg r(y)^{(d-1) / 2} t^{(d+1) / 2} .
$$

To deal with the case $o \notin K_{(n)}$, we observe that there exists a positive constant $\gamma_{5} \in(0,1)$ such that the probability measure of each of the $2^{d}$ coordinate corners of $\mathbb{R}^{d}$ is at least $\gamma_{5}$. If $o \notin K_{(n)}$, then $\left\{x_{1}, \ldots, x_{n}\right\}$ is disjoint from one of these coordinate corners, and hence

$$
\mathbb{P}_{\varrho}\left(o \notin K_{(n)}\right) \leqslant 2^{d}\left(1-\gamma_{5}\right)^{n} .
$$

Now the assertion follows from (4.2)-(4.4).

Subsequently, the estimate of Lemma 4.1 will be used, for instance, to restrict the domain of integration on the right-hand side of (3.2) (cf. Lemma 4.3) and to justify an application of Lebesgue's dominated convergence theorem (see (4.9)). For these applications, we also need that if $c>0$ is such that $\omega:=c \delta^{(d+1) / 2}<1$, then

$$
\int_{0}^{\delta}\left(1-c t^{(d+1) / 2}\right)^{n} d t=\frac{2}{d+1} c^{-2 /(d+1)} \int_{0}^{\omega} s^{2 /(d+1)-1}(1-s)^{n} d s \ll c^{-2 /(d+1)} \cdot n^{-2 /(d+1)},
$$

where we use that $(1-s)^{n} \leqslant e^{-n s}$ for $s \in[0,1]$ and $n \in \mathbb{N}$.

The next lemma allows us to decompose integrals in a suitable way.

Lemma 4.2. If $0 \leqslant t_{0} \leqslant t_{1}<\delta$ and $h: K \rightarrow[0, \infty]$ is a measurable function, then

$$
\int_{K_{t_{0}} \backslash K_{t_{1}}} h(x) d x=\int_{\partial K} \int_{t_{0}}^{t_{1}}(1-t)^{d-1}\langle y, u(y)\rangle h\left(y_{t}\right) d t \mathcal{H}^{d-1}(d y) .
$$

Proof. The map $T: \partial K \times\left[t_{0}, t_{1}\right] \rightarrow K_{t_{0}} \backslash K_{t_{1}}$, with $(y, t) \mapsto(1-t) y$, provides a bilipschitz parametrization of $K_{t_{0}} \backslash K_{t_{1}}$ with $(1-t) y=y_{t} \in \partial K_{t}$. The Jacobian of $T$, for $\mathcal{H}^{d-1}$-almost all $y \in \partial K$ and $t \in\left[t_{0}, t_{1}\right]$, is given by $J T(y, t)=(1-t)^{d-1}\langle y, u(y)\rangle$, where $u(y)$ is the $\left(\mathcal{H}^{d-1}\right.$ almost everywhere) unique exterior unit normal of $\partial K$ at $y$. The assertion now follows from Federer's area/coarea theorem (see [14]). 
In the following, for $\alpha>-1$, we use the important fact that

$$
\int_{\partial K} r(y)^{\alpha} \mathcal{H}^{d-1}(d y)<\infty
$$

which is a result due to Schütt and Werner [41].

By decomposing $\lambda$ into its positive and its negative part, we can henceforth assume that $\lambda$ is a nonnegative integrable function.

Lemma 4.3. As $n$ tends to infinity, we have

$$
\int_{K_{n^{-1 /(d+1)}}} \mathbb{P}_{\varrho}\left(x \notin K_{(n)}\right) \lambda(x) d x=o\left(n^{-2 /(d+1)}\right) .
$$

Proof. Let $\delta>0$ be chosen as in Lemma 4.1 and the subsequent remark. First, we consider a point $x$ in $K_{\delta}$. Let $\omega$ be the minimal distance between the points of $\partial K$ and $K_{\delta}$, and let $z_{1}, \ldots, z_{k}$ be a maximal family of points in $K \backslash \operatorname{int}\left(K_{\delta}\right)$ such that $\left\|z_{i}-z_{j}\right\| \geqslant \frac{\omega}{4}$ for $i \neq j$. We define $p_{0}>0$ by

$$
p_{0}:=\min \left\{\mathbb{P}_{\varrho}\left(z_{i}+\frac{\omega}{4} B^{d}\right): i=1, \ldots, k\right\} .
$$

Let $x \in K_{\delta}$. If $x \notin K_{(n)}$, then there is some $u \in S^{d-1}$ such that $x \in H^{+}(u, t)$ and $K_{(n)} \subset \operatorname{int}\left(H^{-}(u, t)\right)$. Since $x \in K_{\delta}$, we obtain that $K_{(n)} \subset \operatorname{int}\left(H^{-}\left(u, h\left(K_{\delta}, u\right)\right)\right)$. If $z \in$ $H\left(u, h\left(K_{\delta}, u\right)\right) \cap \partial K_{\delta}$, then

$$
z+\frac{\omega}{2} u+\frac{\omega}{2} B^{d} \subset K \cap H^{+}\left(u, h\left(K_{\delta}, u\right)\right) .
$$

By the maximality of the set $\left\{z_{1}, \ldots, z_{k}\right\}$, we have

$$
\left\{z_{1}, \ldots, z_{k}\right\} \cap\left(z+\frac{\omega}{2} u+\frac{\omega}{4} B^{d}\right) \neq \emptyset .
$$

Let $z_{j}$ lie in the intersection. Then $z_{j}+\frac{\omega}{4} B^{d} \subset H^{+}\left(u, h\left(K_{\delta}, u\right)\right)$, and hence $x_{i} \notin z_{j}+\frac{\omega}{4} B^{d}$ for $i=1, \ldots, n$. For $x \in K_{\delta}$, this implies that

$$
\mathbb{P}_{\varrho}\left(x \notin K_{(n)}\right) \leqslant k\left(1-p_{0}\right)^{n} .
$$

Consider $\varepsilon:=\left(2\left(d^{2}-1\right)\right)^{-1}$ and let $n \geqslant \delta^{-(d+1)}$. For $y \in \partial K$ we show that

$$
\int_{n^{-1 /(d+1)}}^{\delta} \mathbb{P}_{\varrho}\left(y_{t} \notin K_{(n)}\right) d t \ll r(y)^{-d /(d+1)} n^{-2 /(d+1)-\varepsilon} .
$$

In fact, if $r(y) \leqslant n^{-(d+1) \varepsilon}$, then Lemma 4.1 and (4.5) yield

$$
\begin{aligned}
\int_{n^{-1 /(d+1)}}^{\delta} \mathbb{P}_{\varrho}\left(y_{t} \notin K_{(n)}\right) d t & \leqslant \int_{0}^{\delta}\left(1-\gamma_{1} r(y)^{(d-1) / 2} t^{(d+1) / 2}\right)^{n} d t \\
& \ll r(y)^{-(d-1) /(d+1)} n^{-2 /(d+1)} \\
& \leqslant r(y)^{-d /(d+1)} n^{-2 /(d+1)-\varepsilon},
\end{aligned}
$$

where the assumption on $r(y)$ is used for the last estimate.

If $r(y) \geqslant n^{-(d+1) \varepsilon}$ and $n \geqslant n_{0}$, where $n_{0}$ depends on $K, \varrho$ and $\lambda$, then Lemma 4.1 implies, for all $t \in\left(n^{-1 /(d+1)}, \delta\right)$, that

$$
\begin{aligned}
\mathbb{P}_{\varrho}\left(y_{t} \notin K_{(n)}\right) & \ll\left(1-\gamma_{1} n^{-\varepsilon\left(d^{2}-1\right) / 2-1 / 2}\right)^{n}=\left(1-\gamma_{1} n^{-3 / 4}\right)^{n} \leqslant e^{-\gamma_{1} n^{1 / 4}} \\
& \leqslant r(K)^{-d /(d+1)} n^{-2 /(d+1)-\varepsilon},
\end{aligned}
$$


which again yields (4.8). In particular, writing $I$ to denote the integral in Lemma 4.3, we obtain from Lemma 4.2, (4.7), (4.8) and (4.6) that

$$
\begin{aligned}
I & \ll \int_{K_{\delta}} \mathbb{P}_{\varrho}\left(x \notin K_{(n)}\right) \lambda(x) d x+\int_{\partial K} \int_{n^{-1 /(d+1)}}^{\delta} \mathbb{P}_{\varrho}\left(y_{t} \notin K_{(n)}\right) d t \mathcal{H}^{d-1}(d y) \\
& \ll k\left(1-p_{0}\right)^{n}+\int_{\partial K} r(y)^{-d /(d+1)} n^{-2 /(d+1)-\varepsilon} \mathcal{H}^{d-1}(d y) \ll n^{-2 /(d+1)-\varepsilon},
\end{aligned}
$$

where we also used the fact that $\lambda$ is integrable on $K$ and bounded on $K \backslash K_{\delta}$. This is the required estimate.

It follows from (3.2), Lemmas 4.3 and 4.2 that

$$
\begin{aligned}
& \lim _{n \rightarrow \infty} n^{2 /(d+1)} \mathbb{E}_{\varrho} \int_{K \backslash K_{(n)}} \lambda(x) d x \\
& \quad=\lim _{n \rightarrow \infty} n^{2 /(d+1)} \int_{K} \mathbb{P}_{\varrho}\left(x \notin K_{(n)}\right) \lambda(x) d x \\
& \quad=\lim _{n \rightarrow \infty} \int_{\partial K} \int_{0}^{n^{-1 /(d+1)}} n^{2 /(d+1)}(1-t)^{d-1}\langle y, u(y)\rangle \mathbb{P}_{\varrho}\left(y_{t} \notin K_{(n)}\right) \lambda\left(y_{t}\right) d t \mathcal{H}^{d-1}(d y) .
\end{aligned}
$$

Lemma 4.1 and (4.5) imply that, if $y \in \partial K$ and $r(y)>0$, then

$$
\int_{0}^{n^{-1 /(d+1)}} n^{2 /(d+1)} \mathbb{P}_{\varrho}\left(y_{t} \notin K_{(n)}\right)\langle y, u(y)\rangle \lambda\left(y_{t}\right) d t \ll r(y)^{-(d-1) /(d+1)} .
$$

Therefore, by (4.6) and since $\lambda$ is bounded and continuous in a neighborhood of $\partial K$, we may apply Lebesgue's dominated convergence theorem, and thus

$$
\lim _{n \rightarrow \infty} n^{2 /(d+1)} \mathbb{E}_{\varrho} \int_{K \backslash K_{(n)}} \lambda(x) d x=\int_{\partial K} \lambda(y) J_{\varrho}(y) \mathcal{H}^{d-1}(d y),
$$

where

$$
J_{\varrho}(y):=\lim _{n \rightarrow \infty} \int_{0}^{n^{-1 /(d+1)}} n^{2 /(d+1)}\langle y, u(y)\rangle \mathbb{P}_{\varrho}\left(y_{t} \notin K_{(n)}\right) d t,
$$

for $\mathcal{H}^{d-1}$-almost all $y \in \partial K$.

Lemma 4.4. If $y \in \partial K$ is a normal boundary point of $K$ with $\kappa(y)=0$, then $J_{\varrho}(y)=0$.

Proof. In view of the estimate (4.4), it is sufficient to prove that, for any given $\varepsilon>0$

$$
\int_{0}^{n^{-1 /(d+1)}} n^{2 /(d+1)} \mathbb{P}_{\varrho}\left(y_{t} \notin K_{(n)}, o \in K_{(n)}\right) d t \ll \varepsilon,
$$

if $n$ is sufficiently large. We choose the coordinate axes in $u(y)^{\perp}$ parallel to the principal curvature directions of $K$ at $y$, and denote by $\Theta_{1}^{\prime}, \ldots, \Theta_{2^{d-1}}^{\prime}$ the corresponding coordinate corners. For $i=1, \ldots, 2^{d-1}$ and $t \in\left(0, n^{-1 /(d+1)}\right)$, let

$$
\Theta_{i, t}:=C(y, t) \cap\left(y_{t}+\left[\Theta_{i}^{\prime}, \mathbb{R}^{+} y\right]\right),
$$

and hence, if $n$ is large enough, then

$$
\int_{\Theta_{i, t}} \varrho(x) d x \gg V\left(\Theta_{i, t}\right),
$$

since $\varrho$ is continuous and positive near $\partial K$. If $y_{t} \notin K_{n}$ and $o \in K_{(n)}$, then there exists a halfspace $H^{-}$that contains $K_{(n)}$ and for which $y_{t} \in \partial H^{-}$. Moreover, for some $i \in\left\{1, \ldots, 2^{d-1}\right\}$ 
the interior of $\mathrm{H}^{-}$is disjoint from $\Theta_{i, t}$. Hence, as in the proof of Lemma 4.1, we have

$$
\mathbb{P}_{\varrho}\left(y_{t} \notin K_{(n)}, o \in K_{(n)}\right) \ll \sum_{i=1}^{2^{d-1}}\left(1-\gamma_{6} V\left(\Theta_{i, t}\right)\right)^{n} .
$$

Since $\partial K$ is twice differentiable in the generalized sense at $y$, we have $r(y)>0$. By assumption, $\kappa(y)=0$; therefore one principal curvature at $y$ is zero, and hence less than $\varepsilon^{d+1} r(y)^{d-2}$. In particular, there exists $\delta^{\prime} \in(0, \delta)$, which by (4.1) depends only on $y$ and $\varepsilon$, such that, if $i \in\left\{1, \ldots, 2^{d-1}\right\}$ and $t \in\left(0, \delta^{\prime}\right)$, then

$$
\mathcal{H}^{d-1}\left(\left(y_{t}+\Theta_{i}^{\prime}\right) \cap K\right) \gg \sqrt{t \varepsilon^{-(d+1)} r(y)^{-(d-2)}} \cdot \sqrt{\operatorname{tr}(y)}^{d-2},
$$

and thus $V\left(\Theta_{i, t}\right) \gg \varepsilon^{-(d+1) / 2} t^{(d+1) / 2}$. Therefore (4.10) follows from (4.5) and (4.11).

Next we consider the case of a normal boundary point $y \in \partial K$ with $\kappa(y)>0$. First, we prove that $J_{\varrho}(y)$ depends only on the random points near $y$ (see Lemma 4.5). In a second step, we compare the simplified expression obtained for $J_{\varrho}(y)$ with the corresponding expression which is obtained if $K$ is a ball.

We start by reparametrizing $y_{t}$ in terms of the probability measure of the corresponding cap. For $t \in\left(0, n^{-1 /(d+1)}\right)$, where $n \geqslant n_{0}$ is sufficiently large so that $\varrho$ is positive and continuous on $C(y, t)$, for all $y \in \partial K$, we consider

$$
\tilde{y}_{s}:=y_{t},
$$

where, for given $s>0$ (sufficiently small), the corresponding $t=t(s)$ is determined by the relation

$$
s=\int_{C(y, t)} \varrho(x) d x .
$$

It is easy to see that the right-hand side of (4.12) is a continuous and strictly increasing function $s=s(t)$ of $t$, if $t>0$ is sufficiently small. This implies that, for a given $s>0$ (sufficiently small), there is a unique $t(s)$ such that (4.12) is satisfied.

Moreover, observe that

$$
\frac{d s}{d t}=\langle u(y), y\rangle \int_{H(y, t) \cap K} \varrho(x) \mathcal{H}^{d-1}(d x)
$$

for $t \in\left(0, n^{-1 /(d+1)}\right)$. We further define

$$
\tilde{C}(y, s):=C(y, t) \text { and } \widetilde{H}(y, s):=\left\{x \in \mathbb{R}^{d}:\langle u(y), x\rangle=\left\langle u(y), \tilde{y}_{s}\right\rangle\right\},
$$

where $t=t(s)$.

Let $Q$ denote the second fundamental form of $\partial K$ at $y$ (cf. (2.1)), considered as a function on $u(y)^{\perp}$. We define

$$
E:=\left\{z \in u(y)^{\perp}: Q(z) \leqslant 1\right\}
$$

and put $u:=u(y)$. Choosing a suitable orthonormal basis $v_{1}, \ldots, v_{d-1}$ of $u(y)^{\perp}$, we have

$$
Q(z)=\sum_{i=1}^{d-1} k_{i}(y) z_{i}^{2}
$$

where $k_{i}(y)$, with $i=1, \ldots, d-1$, are the generalized principal curvatures of $K$ at $y$ and where $z=z_{1} v_{1}+\ldots+z_{d-1} v_{d-1}$. Since $y$ is a normal boundary point of $K$, there is a nondecreasing function $\mu:(0, \infty) \rightarrow \mathbb{R}$ with $\lim _{r \rightarrow 0^{+}} \mu(r)=1$ such that

$$
\frac{\mu(r)^{-1}}{\sqrt{2 r}}(K(u, r)+r u-y) \subset E \subset \frac{\mu(r)}{\sqrt{2 r}}(K(u, r)+r u-y),
$$


where $K(u, r):=K \cap H(u, h(K, u)-r)$. In the following, $\mu_{i}:(0, \infty) \rightarrow \mathbb{R}$, where $i=1,2, \ldots$, always denote nondecreasing functions with $\lim _{r \rightarrow 0^{+}} \mu(r)=1$. Applying (4.14) and Fubini's theorem, we get

$$
V\left(K \cap H^{+}(u, h(K, u)-r)\right)=\mu_{1}(r) \frac{(2 r)^{(d+1) / 2}}{d+1} \alpha_{d-1} \kappa(y)^{-1 / 2},
$$

which yields

$$
s(t)=\mu_{2}(t) \frac{(2 t\langle y, u\rangle)^{(d+1) / 2}}{d+1} \alpha_{d-1} \kappa(y)^{-1 / 2} \varrho(y),
$$

since $\varrho$ is continuous at $y$. Moreover, defining

$$
\eta:=(d+1)^{1 /(d+1)} \alpha_{d-1}^{-1 /(d+1)} \varrho(y)^{-1 /(d+1)} \kappa(y)^{1 /[2(d+1)]},
$$

we obtain

$$
\lim _{s \rightarrow 0^{+}} s^{-1 /(d+1)}\left[(\widetilde{H}(y, s) \cap K)-\tilde{y}_{s}\right]=\eta \cdot E
$$

in the sense of the Hausdorff metric on compact convex sets (see [17] or [35]). Here we also use the fact that

$$
\lim _{s \rightarrow 0^{+}} s^{-1 /(d+1)}\left(\tilde{y}_{s}-\left\langle\tilde{y}_{s}, u\right\rangle u\right)=o .
$$

Now it follows from (4.13) and (4.16) that (4.9) turns into

$$
\begin{aligned}
J_{\varrho}(y)= & (d+1)^{-(d-1) /(d+1)} \alpha_{d-1}^{-2 /(d+1)} \varrho(y)^{-2 /(d+1)} \kappa(y)^{1 /(d+1)} \\
& \times \lim _{n \rightarrow \infty} \int_{0}^{g(n, y)} n^{2 /(d+1)} \mathbb{P}_{\varrho}\left(\tilde{y}_{s} \notin K_{(n)}\right) s^{-(d-1) /(d+1)} d s,
\end{aligned}
$$

where

$$
\lim _{n \rightarrow \infty} n^{1 / 2} g(n, y)=(d+1)^{-1} \alpha_{d-1} \varrho(y)(2\langle u(y), y\rangle)^{(d+1) / 2} \kappa(y)^{-1 / 2} .
$$

The rest of the proof is devoted to identifying the asymptotic behavior of the integral. First, we adjust the domain of integration and the integrand in a suitable way. In a second step, the resulting expression is compared to the case where $K$ is the unit ball. We recall that $x_{1}, \ldots, x_{n}$ are random points in $K$, and we consider $\Xi_{n}:=\left\{x_{1}, \ldots, x_{n}\right\}$, and hence $K_{(n)}=\left[\Xi_{n}\right]$. Let \#X denote the cardinality of a finite set $X \subset \mathbb{R}^{d}$.

Lemma 4.5. For $\varepsilon \in(0,1)$, there exist $\alpha, \beta>1$ and an integer $k>1$, depending only on $\varepsilon$ and $d$, with the following property. If $y \in \partial K$ is a normal boundary point of $K$ with $\kappa(y)>0$ and if $n>n_{0}$, where $n_{0}$ depends on $\varepsilon, y, K, \varrho$, then

$$
\begin{aligned}
\int_{0}^{g(n, y)} \mathbb{P}_{\varrho}\left(\tilde{y}_{s} \notin K_{(n)}\right) s^{-(d-1) /(d+1)} d s= & \int_{\varepsilon^{(d+1) / 2} / n}^{\alpha / n} \varphi(K, y, \varrho, \varepsilon, s) s^{-(d-1) /(d+1)} d s \\
& +O\left(\frac{\varepsilon}{n^{2 /(d+1)}}\right),
\end{aligned}
$$

where

$$
\varphi(K, y, \varrho, \varepsilon, s)=\mathbb{P}_{\varrho}\left(\left(\tilde{y}_{s} \notin\left[\tilde{C}(y, \beta s) \cap \Xi_{n}\right]\right) \text { and }\left(\#\left(\tilde{C}(y, \beta s) \cap \Xi_{n}\right) \leqslant k\right)\right) .
$$

Proof. Let $Q$ be the second fundamental form of $\partial K$ at the normal boundary point $y$, and let $v_{1}, \ldots, v_{d-1}$ be an orthonormal basis of $u(y)^{\perp}$ with respect to $Q$, as described above. Let $\Theta_{1}^{\prime}, \ldots, \Theta_{2^{d-1}}^{\prime}$ be the corresponding coordinate corners and, for $i=1, \ldots, 2^{d-1}$ and for $s \in\left(0, n^{-1 / 2}\right)$, consider

$$
\widetilde{\Theta}_{i, s}:=\tilde{C}(y, s) \cap\left(\tilde{y}_{s}+\left[\Theta_{i}^{\prime}, \mathbb{R}^{+} y\right]\right) .
$$


Let $A_{s}$, with $s>0$, be the affine map of $\mathbb{R}^{d}$ with $A_{s}(y)=y$ for which the associated linear map $\widetilde{A}_{s}$ is determined by $\widetilde{A}_{s}(v)=s^{1 /(d+1)} v$, for $v \in u^{\perp}$, and $\widetilde{A}_{s}(u)=s^{2 /(d+1)} u$. Then $\operatorname{det}\left(\widetilde{A}_{s}\right)=$ $s$ and $A_{s^{-1}}(\tilde{C}(y, s))$ converges in the Hausdorff metric, as $s \rightarrow 0^{+}$, to the cap $\tilde{C}(y)$ of the osculating paraboloid of $K$ at $y$ having volume $\varrho(y)^{-1}$. Here we use the assumptions that $\varrho$ is continuous at $y, \varrho(y)>0$ and relation (4.12) holds. Let $\lambda>0$ be such that $\tilde{y}:=y-\lambda u \in \partial \tilde{C}(y)$. Then $A_{s^{-1}}\left(\widetilde{\Theta}_{i, s}\right)$ converges in the Hausdorff metric, as $s \rightarrow 0^{+}$, to $\tilde{C}(y) \cap\left(\tilde{y}+\left[\Theta_{i}^{\prime}, \mathbb{R}^{+} u\right]\right)$, since (4.17) is satisfied. Since $\varrho$ is continuous and positive at $y$, we thus get

$$
\begin{aligned}
\lim _{s \rightarrow 0^{+}} s^{-1} \int_{\widetilde{\Theta}_{i, s}} \varrho(x) d x & =\lim _{s \rightarrow 0^{+}} s^{-1} V\left(\widetilde{\Theta}_{i, s}\right) \varrho(y) \\
& =\lim _{s \rightarrow 0^{+}} V\left(A_{s^{-1}}\left(\widetilde{\Theta}_{i, s}\right)\right) \varrho(y) \\
& =V\left(\tilde{C}(y) \cap\left(\tilde{y}+\left[\Theta_{i}^{\prime}, \mathbb{R}^{+} u\right]\right)\right) \varrho(y) \\
& =2^{-(d-1)} V(\tilde{C}(y)) \varrho(y) \\
& =2^{-(d-1)} \lim _{s \rightarrow 0^{+}} V\left(A_{s^{-1}}(\tilde{C}(y, s)) \varrho(y)\right. \\
& =2^{-(d-1)} \lim _{s \rightarrow 0^{+}} s^{-1} V(\tilde{C}(y, s)) \varrho(y) \\
& =2^{-(d-1)} \lim _{s \rightarrow 0^{+}} s^{-1} \int_{\tilde{C}(y, s)} \varrho(x) d x \\
& =2^{-(d-1)},
\end{aligned}
$$

that is,

$$
\lim _{s \rightarrow 0^{+}} s^{-1} \int_{\widetilde{\Theta}_{i, s}} \varrho(x) d x=2^{-(d-1)} .
$$

Let $\alpha>1$ be chosen such that

$$
2^{d-1+2 d /(d+1)} \int_{2^{-d} \alpha}^{\infty} e^{-x} x^{2 /(d+1)-1} d x \leqslant \varepsilon .
$$

Then we first choose $\beta \geqslant(16(d-1))^{d+1}$ such that

$$
2^{d-1} e^{-d^{-1} 2^{-(d+3)} \beta^{1 /(d+1)} \varepsilon^{(d+1) / 2}} \leqslant \frac{\varepsilon}{\alpha^{2 /(d+1)}},
$$

and then we fix an integer $k>1$ such that

$$
\frac{(\alpha \beta)^{k}}{k !} \leqslant \frac{\varepsilon}{\alpha^{2 /(d+1)}}
$$

Lemma 4.5 follows from the following three statements, which we will prove assuming that $n$ is sufficiently large:

(i)

$$
\begin{aligned}
& \int_{0}^{g(n, y)} \mathbb{P}_{\varrho}\left(\tilde{y}_{s} \notin K_{(n)}\right) s^{-(d-1) /(d+1)} d s \\
& \quad=\int_{\varepsilon^{(d+1) / 2} / n}^{\alpha / n} \mathbb{P}_{\varrho}\left(\tilde{y}_{s} \notin K_{(n)}\right) s^{-(d-1) /(d+1)} d s+O\left(\frac{\varepsilon}{n^{2 /(d+1)}}\right) ;
\end{aligned}
$$

(ii) if $\varepsilon^{(d+1) / 2} / n<s<\alpha / n$, then

$$
\mathbb{P}_{\varrho}\left(\#\left(\tilde{C}(y, \beta s) \cap \Xi_{n}\right) \geqslant k\right)=O\left(\frac{\varepsilon}{\alpha^{2 /(d+1)}}\right) ;
$$

(iii) if $\varepsilon^{(d+1) / 2} / n<s<\alpha / n$, then

$$
\mathbb{P}_{\varrho}\left(\tilde{y}_{s} \notin K_{(n)}\right)=\mathbb{P}_{\varrho}\left(\tilde{y}_{s} \notin\left[\tilde{C}(y, \beta s) \cap \Xi_{n}\right]\right)+O\left(\frac{\varepsilon}{\alpha^{2 /(d+1)}}\right) .
$$


To prove (i), we first observe that

$$
\int_{0}^{\varepsilon^{(d+1) / 2} / n} \mathbb{P}_{\varrho}\left(\tilde{y}_{s} \notin K_{(n)}\right) s^{-(d-1) /(d+1)} d s \leqslant \int_{0}^{\varepsilon^{(d+1) / 2} / n} s^{-(d-1) /(d+1)} d s \ll \frac{\varepsilon}{n^{2 /(d+1)}} .
$$

If $\alpha / n<s<g(n, y), o \in K_{(n)}, \tilde{y}_{s} \notin K_{(n)}$ and if $n$ is sufficiently large, then there is some $i \in$ $\left\{1, \ldots, 2^{d-1}\right\}$ such that $\widetilde{\Theta}_{i, s} \cap K_{(n)}=\emptyset$, and hence (4.4) and (4.18) yield

$$
\mathbb{P}_{\varrho}\left(\tilde{y}_{s} \notin K_{(n)}\right) \ll 2^{d-1}\left(1-2^{-d} s\right)^{n} \leqslant 2^{d-1} e^{-2^{-d} n s} .
$$

Therefore, by the definition of $\alpha$, we get

$$
\begin{aligned}
\int_{\alpha / n}^{g(n, y)} \mathbb{P}_{\varrho}\left(\tilde{y}_{s} \notin K_{(n)}\right) s^{-(d-1) /(d+1)} d s & \ll 2^{d-1} \int_{\alpha / n}^{\infty} e^{-2^{-d} n s} s^{2 /(d+1)-1} d s \\
& =2^{d-1} 2^{2 d /(d+1)} n^{-2 /(d+1)} \int_{2^{-d} \alpha}^{\infty} e^{-x} x^{2 /(d+1)-1} d x \\
& \leqslant \varepsilon n^{-2 /(d+1)},
\end{aligned}
$$

which verifies (i).

Next (ii) simply follows from (4.12) as, if $s<\alpha / n$, then

$$
\mathbb{P}_{\varrho}\left(\#\left(\tilde{C}(y, \beta s) \cap \Xi_{n}\right) \geqslant k\right)=\left(\begin{array}{l}
n \\
k
\end{array}\right)(\beta s)^{k} \leqslant\left(\begin{array}{l}
n \\
k
\end{array}\right)\left(\frac{\alpha \beta}{n}\right)^{k}<\frac{(\alpha \beta)^{k}}{k !} \leqslant \frac{\varepsilon}{\alpha^{2 /(d+1)}} .
$$

Now we prove (iii). To this end, for $s$ in the given range, our plan is to construct sets $\widetilde{\Omega}_{1, s}, \ldots, \widetilde{\Omega}_{2^{d-1}, s} \subset K$ such that

$$
\int_{\widetilde{\Omega}_{i, s}} \varrho(x) d x \geqslant d^{-1} 2^{-(d+3)} \beta^{1 /(d+1)} s \quad \text { for } \quad i=1, \ldots, 2^{d-1},
$$

and if $\tilde{y}_{s} \in K_{(n)}$ but $\tilde{y}_{s} \notin\left[\tilde{C}(y, \beta s) \cap \Xi_{n}\right]$, then $\Xi_{n} \cap \widetilde{\Omega}_{i, s}=\emptyset$ for some $i \in\left\{1, \ldots, 2^{d-1}\right\}$.

For $i=1, \ldots, 2^{d-1}$, let $w_{i} \in \Theta_{i}^{\prime}$ be the vector whose coordinates (up to sign) in the basis $v_{1}, \ldots, v_{d-1}$ are

$$
w_{i}:=(\sqrt{\beta} s)^{1 /(d+1)} \frac{\eta}{2 \sqrt{d-1}}\left( \pm \frac{1}{\sqrt{k_{1}(y)}}, \ldots, \pm \frac{1}{\sqrt{k_{d-1}(y)}}\right) .
$$

Further, for $i=1, \ldots, 2^{d-1}$ we define

$$
\widetilde{\Omega}_{i, s}=\left[\tilde{y}_{\sqrt{\beta} s}+w_{i}, K \cap\left(\tilde{y}_{s}+\Theta_{i}^{\prime}\right)\right] .
$$

Then, if $s>0$ is small enough, we have $\tilde{y}_{\sqrt{\beta} s}+w_{i} \in K$, and hence $\widetilde{\Omega}_{i, s} \subset K$. Here we use the fact that

$$
w_{i} \in(\sqrt{\beta} s)^{1 /(d+1)} \frac{1}{2} \eta E
$$

and therefore, by (4.16), we have

$$
\tilde{y}_{\sqrt{\beta} s}+w_{i} \in \widetilde{H}(y, \sqrt{\beta} s) \cap K \subset K .
$$

Recall that $\tilde{y}_{s}=(1-t) y$, where $s$ and $t$ are related by (4.15). Hence, if $s, t>0$ are sufficiently small, then

$$
\left\langle u(y), \tilde{y}_{s}-\tilde{y}_{\sqrt{\beta}}\right\rangle>\frac{\beta^{1 /(d+1)}-1}{2}\left\langle u(y), y-\tilde{y}_{s}\right\rangle>\frac{\beta^{1 /(d+1)}}{4}\left\langle u(y), y-\tilde{y}_{s}\right\rangle,
$$

since $\beta \geqslant 2^{d+1}$; moreover, we have

$$
\left\langle u(y), y-\tilde{y}_{s}\right\rangle \cdot \mathcal{H}^{d-1}\left(K \cap\left(\tilde{y}_{s}+\Theta_{i}^{\prime}\right)\right) \geqslant V\left(\widetilde{\Theta}_{i, s}\right) .
$$


Combining (4.21), (4.22) and (4.18) together with the continuity of $\varrho$ at $y$ with $\varrho(y)>0$, we get

$$
\begin{aligned}
\int_{\tilde{\Omega}_{i, s}} \varrho(x) d x & \geqslant \frac{1}{\sqrt{2}} \frac{1}{d} \varrho(y)\left\langle u(y), \tilde{y}_{s}-\tilde{y}_{\sqrt{\beta} s}\right\rangle \mathcal{H}^{d-1}\left(K \cap\left(\tilde{y}_{s}+\Theta_{i}^{\prime}\right)\right) \\
& \geqslant \frac{\beta^{1 /(d+1)}}{4} \frac{1}{\sqrt{2} d} V\left(\widetilde{\Theta}_{i, s}\right) \\
& \geqslant \frac{\beta^{1 /(d+1)}}{4} \frac{1}{2 d} \int_{\tilde{\Theta}_{i, s}} \varrho(x) d x \\
& \geqslant \frac{\beta^{1 /(d+1)} s}{8 d 2^{d}}
\end{aligned}
$$

which proves (4.20).

It is still left to prove that, if $\tilde{y}_{s} \in K_{(n)}$ but $\tilde{y}_{s} \notin\left[\tilde{C}(y, \beta s) \cap \Xi_{n}\right]$, then $\Xi_{n} \cap \widetilde{\Omega}_{i, s}=\emptyset$ for some $i \in\left\{1, \ldots, 2^{d-1}\right\}$. Hence we assume that $\tilde{y}_{s} \in K_{(n)}$ but $\tilde{y}_{s} \notin\left[\tilde{C}(y, \beta s) \cap \Xi_{n}\right]$. Then there exist $a \in\left[\tilde{C}(y, \beta s) \cap \Xi_{n}\right]$ and $b \in K_{(n)} \backslash \tilde{C}(y, \beta s)$ such that $\tilde{y}_{s} \in[a, b]$, and hence there exists a hyperplane $H$ containing $\tilde{y}_{s}$ and bounding the halfspaces $H^{+}$and $H^{-}$such that $\tilde{C}(y, \beta s) \cap$ $\Xi_{n} \subset \operatorname{int}\left(H^{+}\right)$and $b \in \operatorname{int}\left(H^{-}\right)$.

Next we show that there exists $q \in\left[\tilde{y}_{s}, b\right]$ such that

$$
q \in H^{-} \cap\left(\tilde{y}_{\sqrt{\beta} s}+\frac{\eta}{2 \sqrt{d-1}}(\sqrt{\beta} s)^{1 /(d+1)} E\right) .
$$

In fact, define $q:=\left[\tilde{y}_{s}, b\right] \cap \widetilde{H}(y, \sqrt{\beta} s)$ and $q^{\prime}:=\left[\tilde{y}_{s}, b\right] \cap \widetilde{H}(y, \beta s)$. Since $a \in H^{+}$and $\tilde{y}_{s} \in H$, it follows that $q \in H^{-}$. From (4.16) we get

$$
\widetilde{H}(y, \beta s) \cap K \subset \tilde{y}_{\beta s}+2 \beta^{1 /(d+1)} s^{1 /(d+1)} \eta E .
$$

By (4.15), we have

$$
\begin{aligned}
\left\langle u(y), \tilde{y}_{s}-\tilde{y}_{\beta s}\right\rangle & <\frac{\beta^{2 /(d+1)}}{\beta^{2 /(d+1)}-1} \cdot \frac{\beta^{2 /(d+1)}-1}{\beta^{2 /(d+1)}-\beta^{1 /(d+1)}}\left\langle u(y), \tilde{y}_{\sqrt{\beta} s}-\tilde{y}_{\beta s}\right\rangle \\
& <\frac{\beta^{1 /(d+1)}}{\beta^{1 /(d+1)}-1}\left\langle u(y), \tilde{y}_{\sqrt{\beta} s}-\tilde{y}_{\beta s}\right\rangle .
\end{aligned}
$$

Furthermore, by elementary geometry we have

$$
\frac{\left\|q-\tilde{y}_{\sqrt{\beta} s}\right\|}{\left\|q^{\prime}-\tilde{y}_{\beta s}\right\|}=\frac{\left\langle u, \tilde{y}_{s}-\tilde{y}_{\sqrt{\beta} s}\right\rangle}{\left\langle u, \tilde{y}_{s}-\tilde{y}_{\beta s}\right\rangle} .
$$

Then, by (4.24) and (4.25), we have

$$
\begin{aligned}
q & \in \tilde{y}_{\sqrt{\beta} s}+\frac{\left\langle u, \tilde{y}_{s}-\tilde{y}_{\sqrt{\beta} s}\right\rangle}{\left\langle u, \tilde{y}_{s}-\tilde{y}_{\beta s}\right\rangle} \cdot 2(\beta s)^{1 /(d+1)} \eta E \\
& \subset \tilde{y}_{\sqrt{\beta} s}+\left(1-\frac{\left\langle u, \tilde{y}_{\sqrt{\beta} s}-\tilde{y}_{\beta s}\right\rangle}{\left\langle u, \tilde{y}_{s}-\tilde{y}_{\beta s}\right\rangle}\right) \cdot 2 \beta^{1 /(d+1)} s^{1 /(d+1)} \eta E \\
& \subset \tilde{y}_{\sqrt{\beta} s}+2 s^{1 /(d+1)} \eta E \\
& \subset \tilde{y}_{\sqrt{\beta} s}+\frac{1}{2 \sqrt{d-1}}(\sqrt{\beta} s)^{1 /(d+1)} \eta E
\end{aligned}
$$

where $\beta \geqslant(16(d-1))^{d+1}$ is used for the last inclusion. Now there exists some $i \in\left\{1, \ldots, 2^{d-1}\right\}$ such that $\tilde{y}_{s}+\Theta_{i}^{\prime} \subset H^{-}$, and hence $q+\Theta_{i}^{\prime} \subset H^{-}$. By (4.23) this finally yields

$$
\tilde{y}_{\sqrt{\beta} s}+w_{i} \subset q+\Theta_{i}^{\prime} \subset H^{-} \text {. }
$$

Therefore, we obtain that $\widetilde{\Omega}_{i, s} \cap \Xi_{n}=\emptyset$. 
Finally, (iii) follows since, if $\varepsilon^{(d+1) / 2} / n<s<\alpha / n$, then

$$
\begin{aligned}
0 & \leqslant \mathbb{P}_{\varrho}\left(\tilde{y}_{s} \notin\left[\tilde{C}(y, \beta s) \cap \Xi_{n}\right]\right)-\mathbb{P}_{\varrho}\left(\tilde{y}_{s} \notin K_{(n)}\right) \\
& \leqslant \sum_{i=1}^{2^{d-1}}\left(1-\int_{\tilde{\Omega}_{i, s}} \varrho(x) d x\right)^{n} \\
& \leqslant \sum_{i=1}^{2^{d-1}} e^{-n \int_{\tilde{\Omega}_{i, s}} \varrho(x) d x} \\
& \leqslant 2^{d-1} e^{-d^{-1} 2^{-(d+3)} \beta^{1 /(d+1)} \varepsilon^{(d+1) / 2}} \\
& \leqslant \varepsilon \alpha^{-2 /(d+1)}
\end{aligned}
$$

by the choice of $\beta$.

REMARK. As a consequence of the proof of Lemma 4.5, it follows that

$$
\begin{aligned}
J_{\varrho}(y)= & (d+1)^{-(d-1) /(d+1)} \alpha_{d-1}^{-2 /(d+1)} \varrho(y)^{-2 /(d+1)} \kappa(y)^{1 /(d+1)} \\
& \times \lim _{n \rightarrow \infty} \int_{0}^{n^{-1 / 2}} n^{2 /(d+1)} \mathbb{P}_{\varrho}\left(\tilde{y}_{s} \notin K_{(n)}\right) s^{-(d-1) /(d+1)} d s .
\end{aligned}
$$

In fact, since $g(n, y) \ll n^{-1 / 2}$, it is sufficient to show that

$$
\lim _{n \rightarrow \infty} n^{2 /(d+1)} \int_{c_{1} n^{-1 / 2}}^{c_{2} n^{-1 / 2}} \mathbb{P}_{\varrho}\left(\tilde{y}_{s} \notin K_{(n)}\right) s^{-(d-1) /(d+1)} d s=0
$$

for any two constants $0<c_{1} \leqslant c_{2}<\infty$. Since the estimate (4.19) can be applied, we get

$$
\begin{aligned}
n^{2 /(d+1)} \int_{c_{1} n^{-1 / 2}}^{c_{2} n^{-1 / 2}} \mathbb{P}_{\varrho}\left(\tilde{y}_{s} \notin K_{(n)}\right) s^{-(d-1) /(d+1)} d s & \ll n^{2 /(d+1)} \int_{c_{1} n^{-1 / 2}}^{c_{2} n^{-1 / 2}} e^{-2^{-d} n s} s^{2 /(d+1)-1} d s \\
& \ll \int_{2^{-d} c_{1} n^{1 / 2}}^{2^{-d} c_{2} n^{1 / 2}} e^{-r} r^{2 /(d+1)-1} d r,
\end{aligned}
$$

from which the conclusion follows.

Subsequently, we write $\mathbf{1}$ to denote the constant one function on $\mathbb{R}^{d}$. For the unit ball $B^{d}$, we recall that $B_{(n)}^{d}$ denotes the convex hull of $n$ random points distributed uniformly and independently in $B^{d}$. We fix a point $w \in \partial B^{d}$ and, for $s \in\left(0, \frac{1}{2}\right)$, define $\tilde{w}_{s}:=t \cdot w$, where $t \in(0,1)$ is chosen such that

$$
s=\alpha_{d}^{-1} \cdot V\left(\left\{x \in B^{d}:\langle x, w\rangle \geqslant\left\langle\tilde{w}_{s}, w\right\rangle\right\}\right) .
$$

By a classical result due to Wieacker $[49]$, we have

$$
\lim _{n \rightarrow \infty} n^{2 /(d+1)} \mathbb{E}_{1, B^{d}} V\left(B^{d} \backslash B_{(n)}^{d}\right)=c_{d} \omega_{d} \alpha_{d}^{2 /(d+1)},
$$

where the constant $c_{d}$ is given in (2.2). Hence, it follows from (4.9), (4.26) and the preceding remark that

$$
\lim _{n \rightarrow \infty} \int_{0}^{n^{-1 / 2}} n^{2 /(d+1)} \mathbb{P}_{\mathbf{1}, B^{d}}\left(\tilde{w}_{s} \notin B_{(n)}^{d}\right) s^{-(d-1) /(d+1)} d s=c_{d}(d+1)^{(d-1) /(d+1)} \alpha_{d-1}^{2 /(d+1)} .
$$

We are now going to show that the same limit is obtained if $B^{d}$ is replaced by the convex body $K$ and if a normal boundary point $y$ of $K$ with positive Gauss curvature is considered instead of $w \in \partial B^{d}$. 
Lemma 4.6. If $y \in \partial K$ is a normal boundary point of $K$ satisfying $\kappa(y)>0$, then

$$
\lim _{n \rightarrow \infty} \int_{0}^{n^{-1 / 2}} n^{2 /(d+1)} \mathbb{P}_{\varrho}\left(\tilde{y}_{s} \notin K_{(n)}\right) s^{-(d-1) /(d+1)} d s=c_{d}(d+1)^{(d-1) /(d+1)} \alpha_{d-1}^{2 /(d+1)} .
$$

Proof. Let $\varepsilon \in(0,1)$ be arbitrarily chosen. According to Lemma 4.5 and its notation, and by the preceding remark, if $n$ is sufficiently large, we have

$$
\begin{aligned}
\int_{0}^{n^{-1 / 2}} & \mathbb{P}_{\varrho}\left(\tilde{y}_{s} \notin K_{(n)}\right) s^{-(d-1) /(d+1)} d s \\
= & O\left(\frac{\varepsilon}{n^{2 /(d+1)}}\right)+\sum_{i=0}^{k}\left(\begin{array}{c}
n \\
i
\end{array}\right) \int_{\varepsilon^{(d+1) / 2} / n}^{\alpha / n}(\beta s)^{i}(1-\beta s)^{n-i} \\
& \times \mathbb{P}_{\varrho, \tilde{C}(y, \beta s)}\left(\tilde{y}_{s} \notin \tilde{C}(y, \beta s)_{(i)}\right) s^{-(d-1) /(d+1)} d s .
\end{aligned}
$$

We fix a unit vector $p$, and consider the reference paraboloid $\Psi$ which is the graph of $z \mapsto\|z\|^{2}$ on $p^{\perp}$. For $\tau>0$, define

$$
C(\tau):=\left\{z+t p: z \in p^{\perp} \text { and }\|z\|^{2} \leqslant t \leqslant \tau^{2 /(d+1)}\right\},
$$

that is, a cap of $\Psi$ of height $\tau^{2 /(d+1)}$. It is easy to check that $V(C(\tau))=\tau V(C(1))$. We define

$$
\tilde{s}(\beta, s):=\frac{V(\tilde{C}(y, \beta s))}{V(C(\beta))} .
$$

Then (4.12) implies that

$$
\tilde{s}(\beta, s)=\frac{\beta s}{\mu(\beta, s) \varrho(y) \beta V(C(1))}=\frac{s}{\mu(\beta, s) \varrho(y) V(C(1))},
$$

where $\mu(\beta, s) \rightarrow 1$ as $s \rightarrow 0^{+}$. Let $A_{s}$, with $s>0$, denote the affinity of $\mathbb{R}^{d}$ with $A_{s}(y)=y$ for which the associated linear map $\tilde{A}_{s}$ satisfies $\tilde{A}_{s}(v)=s^{1 /(d+1)} v$ for $v \in u^{\perp}$ and $\tilde{A}_{s}(u)=$ $s^{2 /(d+1)} u$. Then the image under $A_{s^{-1}}$ of a cap of $K$ at $y$ converges in the Hausdorff metric, as $s \rightarrow 0^{+}$, to a cap of the osculating paraboloid of $K$ at $y$. For a more explicit statement, let $A$ be a volume-preserving affinity of $\mathbb{R}^{d}$ such that $A(y)=o$ and $A(y-u)=p$, which maps the osculating paraboloid of $K$ at $y$ to $\Psi$. Then $\Phi_{s, \beta}:=A \circ A_{\tilde{s}(\beta, s)^{-1}}$ is an affinity satisfying

$$
\Phi_{s, \beta}(y)=o, \quad \operatorname{det}\left(\Phi_{s, \beta}\right)=\tilde{s}(\beta, s)^{-1}=\frac{V(C(\beta))}{V(\tilde{C}(y, \beta s))},
$$

and, consequently, $\Phi_{s, \beta}(\tilde{C}(y, \beta s)) \rightarrow C(\beta)$ in the Hausdorff metric as $s \rightarrow 0^{+}$. Moreover, we have

$$
\lim _{s \rightarrow 0^{+}} \Phi_{s, \beta}\left(\tilde{y}_{s}\right)=\lim _{s \rightarrow 0^{+}} \Phi_{s, 1}\left(\tilde{y}_{s}\right)=p,
$$

since $\mu(\beta, s) \rightarrow 1$ and $\mu(1, s) \rightarrow 1$ as $s \rightarrow 0^{+}, \tilde{y}_{s} \in \partial \tilde{C}(y, s)$ and $\Phi_{s, 1}\left(\tilde{y}_{s}\right) \in \partial C(1)$, and by (4.17). Since $\varrho$ is continuous at $y$, for $i=0, \ldots, k$, the properties of $\Phi_{s, \beta}$ imply that

$$
\lim _{s \rightarrow 0^{+}} \mathbb{P}_{\varrho, \tilde{C}(y, \beta s)}\left(\tilde{y}_{s} \notin \tilde{C}(y, \beta s)_{(i)}\right)=\mathbb{P}_{\mathbf{1}, C(\beta)}\left(p \notin C(\beta)_{(i)}\right) .
$$

From (4.28) and (4.29) we get

$$
\begin{aligned}
\int_{0}^{n^{-1 / 2}} \mathbb{P}_{\varrho}\left(\tilde{y}_{s} \notin K_{(n)}\right) s^{-(d-1) /(d+1)} d s=O\left(\frac{\varepsilon}{n^{2 /(d+1)}}\right)+\sum_{i=0}^{k}\left(\begin{array}{c}
n \\
i
\end{array}\right) \int_{\varepsilon^{(d+1) / 2} / n}^{\alpha / n}(\beta s)^{i}(1-\beta s)^{n-i} \\
\times \mathbb{P}_{\mathbf{1}, C(\beta)}\left(p \notin C(\beta)_{(i)}\right) s^{-(d-1) /(d+1)} d s .
\end{aligned}
$$


The same formula is obtained for

$$
\int_{0}^{n^{-1 / 2}} \mathbb{P}_{\mathbf{1}, B^{d}}\left(\tilde{w}_{s} \notin B_{(n)}^{d}\right) s^{-(d-1) /(d+1)} d s,
$$

since $C(\beta)$ is independent of $K$. Since $\varepsilon \in(0,1)$ was arbitrary, it follows that

$$
\begin{aligned}
& \lim _{n \rightarrow \infty} \int_{0}^{n^{-1 / 2}} n^{2 /(d+1)} \mathbb{P}_{\varrho}\left(\tilde{y}_{s} \notin K_{(n)}\right) s^{-(d-1) /(d+1)} d s \\
& \quad=\lim _{n \rightarrow \infty} \int_{0}^{n^{-1 / 2}} n^{2 /(d+1)} \mathbb{P}_{\mathbf{1}, B^{d}}\left(\tilde{w}_{s} \notin B_{(n)}^{d}\right) s^{-(d-1) /(d+1)} d s .
\end{aligned}
$$

Now (4.27) yields Lemma 4.6.

Proof of Theorem 3.1. Let $y \in \partial K$ be a normal boundary point of $K$. Combining Lemma 4.4, Lemma 4.6 and (4.26), we obtain

$$
J_{\varrho}(y)=c_{d} \varrho(y)^{-2 /(d+1)} \kappa(y)^{1 /(d+1)} .
$$

Therefore Theorem 3.1 is implied by (4.9).

\section{Polarity and the proof of Theorem 2.1}

In this section, we deduce Theorem 2.1 and Theorem 2.2 from Theorem 3.1 and Corollary 3.2, respectively. In order to obtain more general results, for not necessarily homogeneous or isotropic hyperplane distributions, we start with a description of the basic setting.

Let $K \subset \mathbb{R}^{d}$ be a convex body with $o \in \operatorname{int}(K)$, let $K^{*}:=\left\{z \in \mathbb{R}^{d}:\langle x, z\rangle \leqslant 1\right.$ for all $x \in$ $K\}$ denote the polar body of $K$ and consider $K_{1}:=K+B^{d}$. Let $\mathcal{H}_{K}$ denote the set of all hyperplanes $H$ in $\mathbb{R}^{d}$ for which $H \cap \operatorname{int}(K)=\emptyset$ and $H \cap K_{1} \neq \emptyset$. The motion invariant locally finite measure $\mu$ on the space $A(d, d-1)$ of hyperplanes, which satisfies $\mu\left(\mathcal{H}_{K}\right)=2$, is explicitly given by

$$
\mu=2 \int_{S^{d-1}} \int_{0}^{\infty} \mathbf{1}\{H(u, t) \in \cdot\} d t \sigma(d u),
$$

where $\sigma$ is the rotation invariant probability measure on the unit sphere $S^{d-1}$. The model of a random polytope (random polyhedral set) described in the introduction is based on random hyperplanes with distribution $\mu_{K}:=2^{-1}\left(\mu\left\llcorner\mathcal{H}_{K}\right)\right.$. More generally, we now consider random hyperplanes with distribution

$$
\mu_{q}:=\int_{S^{d-1}} \int_{0}^{\infty} \mathbf{1}\{H(u, t) \in \cdot\} q(t, u) d t \sigma(d u)
$$

where $q:[0, \infty) \times S^{d-1} \rightarrow[0, \infty)$ is a measurable function which has the following properties:

(q1) it is concentrated on $D_{K}:=\left\{(t, u) \in[0, \infty) \times S^{d-1}: h(K, u) \leqslant t \leqslant h\left(K_{1}, u\right)\right\}$;

(q2) it is positive and continuous in a neighborhood of $\left\{(t, u) \in[0, \infty) \times S^{d-1}: t=h(K, u)\right\}$ relative to $D_{K}$;

(q3) it satisfies $\mu_{q}\left(\mathcal{H}_{K}\right)=1$.

The intersection of $n$ halfspaces $H_{i}^{-}$containing the origin $o$ and bounded by $n$ independent random hyperplanes $H_{i}$ with distribution $\mu_{q}$ is denoted by $K^{(n)}:=\bigcap_{i=1}^{n} H_{i}^{-}$. Probabilities and expectations with respect to $\mu_{q}$ are denoted by $\mathbb{P}_{\mu_{q}}$ and $\mathbb{E}_{\mu_{q}}$, respectively. The special example $q \equiv \mathbf{1}_{D_{K}}\left(q\right.$ is the characteristic function of $D_{K}$ ) covers the situation discussed in the introduction.

In the following, as well as the support function, we also need the radial function $\rho(L, \cdot)$ of a convex body $L$ with $o \in \operatorname{int}(L)$. Let $F$ be a nonnegative measurable functional on convex 
polyhedral sets in $\mathbb{R}^{d}$. Using (5.1) and Fubini's theorem, we get

$$
\begin{aligned}
\mathbb{E}_{\mu_{q}}\left(F\left(K^{(n)}\right)\right)= & \int_{A(d, d-1)^{n}} F\left(\bigcap_{i=1}^{n} H_{i}^{-}\right) \mu_{q}^{\otimes n}\left(d\left(H_{1}, \ldots, H_{n}\right)\right) \\
= & \int_{\left(S^{d-1}\right)^{n}} \int_{h\left(K, u_{1}\right)}^{h\left(K_{1}, u_{1}\right)} \ldots \int_{h\left(K, u_{n}\right)}^{h\left(K_{1}, u_{n}\right)} F\left(\bigcap_{i=1}^{n} H_{i}^{-}\left(u_{i}, t_{i}\right)\right) \prod_{i=1}^{n} q\left(t_{i}, u_{i}\right) \\
& \times d t_{n} \ldots d t_{1} \sigma^{\otimes n}\left(d\left(u_{1}, \ldots, u_{n}\right)\right) .
\end{aligned}
$$

For $t_{1}, \ldots, t_{n}>0$, we have

$$
\bigcap_{i=1}^{n} H_{i}^{-}\left(u_{i}, t_{i}\right)=\left[t_{1}^{-1} u_{1}, \ldots, t_{n}^{-1} u_{n}\right]^{*} .
$$

Using the substitution $s_{i}=1 / t_{i}, \rho\left(L^{*}, u_{i}\right)=h\left(L, u_{i}\right)^{-1}$ for $L \in \mathcal{K}^{n}$ with $o \in \operatorname{int}(L)$, and polar coordinates, we obtain

$$
\mathbb{E}_{\mu_{q}}\left(F\left(K^{(n)}\right)\right)=\frac{1}{\omega_{d}^{n}} \int_{\left(K^{*} \backslash K_{1}^{*}\right)^{n}} F\left(\left[x_{1}, \ldots, x_{n}\right]^{*}\right) \prod_{i=1}^{n}\left(\tilde{q}\left(x_{i}\right)\left\|x_{i}\right\|^{-(d+1)}\right) d\left(x_{1}, \ldots, x_{n}\right),
$$

with $K_{1}^{*}:=\left(K_{1}\right)^{*}$ and

$$
\tilde{q}(x):=q\left(\frac{1}{\|x\|}, \frac{x}{\|x\|}\right), \quad x \in K^{*} \backslash\{o\} .
$$

The case $n=1$ and $F \equiv 1$ yields

$$
\frac{1}{\omega_{d}} \int_{K^{*} \backslash K_{1}^{*}} \tilde{q}(x)\|x\|^{-(d+1)} d x=1
$$

and hence

$$
\varrho(x):= \begin{cases}\omega_{d}^{-1} \tilde{q}(x)\|x\|^{-(d+1)}, & x \in K^{*} \backslash K_{1}^{*}, \\ 0, & x \in K_{1}^{*},\end{cases}
$$

is a probability density with respect to $\mathcal{H}^{d}\left\llcorner K^{*}\right.$ that is positive and continuous in a neighborhood of $\partial K^{*}$ relative to $K^{*}$. Thus, we have

$$
\begin{aligned}
\mathbb{E}_{\mu_{q}}\left(F\left(K^{(n)}\right)\right) & =\int_{\left(K^{*}\right)^{n}} F\left(\left[x_{1}, \ldots, x_{n}\right]^{*}\right) \prod_{i=1}^{n} \varrho\left(x_{i}\right) d\left(x_{1}, \ldots, x_{n}\right) \\
& =\mathbb{E}_{\varrho, K^{*}}\left(F\left(\left(K_{(n)}^{*}\right)^{*}\right)\right)
\end{aligned}
$$

where $K_{(n)}^{*}:=\left(K^{*}\right)_{(n)}$.

Proposition 5.1. Let $K \subset \mathbb{R}^{d}$ be a convex body with $o \in \operatorname{int}(K)$, and let $q$ and $\varrho$ be defined as above. Then the random polyhedral sets $K^{(n)}$ and $\left(K_{(n)}^{*}\right)^{*}$ are equal in distribution.

For a first application, let

$$
F(P):=\mathbf{1}\left\{P \subset K_{1}\right\}(W(P)-W(K)),
$$

for a polyhedral set $P \subset \mathbb{R}^{d}$, with the convention $0 \cdot \infty:=0$. For $x_{1}, \ldots, x_{n} \in K^{*} \backslash K_{1}^{*}$, we have $K \subset\left[x_{1}, \ldots, x_{n}\right]^{*}$ and, arguing as before, we have

$$
\begin{aligned}
F\left(\left[x_{1}, \ldots, x_{n}\right]^{*}\right) & =\mathbf{1}\left\{\left[x_{1}, \ldots, x_{n}\right]^{*} \subset K_{1}\right\}\left(W\left(\left[x_{1}, \ldots, x_{n}\right]^{*}\right)-W(K)\right) \\
& =2 \cdot \mathbf{1}\left\{\left[x_{1}, \ldots, x_{n}\right]^{*} \subset K_{1}\right\} \int_{K^{*} \backslash\left[x_{1}, \ldots, x_{n}\right]}^{\lambda(x) d x,}
\end{aligned}
$$


where

$$
\lambda(x):= \begin{cases}\omega_{d}^{-1}\|x\|^{-(d+1)}, & x \in K^{*} \backslash K_{1}^{*}, \\ 0, & x \in K_{1}^{*} .\end{cases}
$$

Note that if $\left[x_{1}, \ldots, x_{n}\right]^{*} \subset K_{1}$, then the set $\left[x_{1}, \ldots, x_{n}\right]^{*}$ is bounded; hence $o \in$ $\operatorname{int}\left(\left[x_{1}, \ldots, x_{n}\right]\right)$, and therefore $K_{1}^{*} \subset\left[x_{1}, \ldots, x_{n}\right]^{* *}=\left[x_{1}, \ldots, x_{n}\right]$.

As in [9], it can be shown that $\mathbb{P}_{\mu_{q}}\left(K^{(n)} \not \subset K_{1}\right) \ll \alpha^{n}$, for some $\alpha \in(0,1)$ depending on $K$ and $q$. By Proposition 5.1, we also get

$$
\mathbb{P}_{\varrho, K^{*}}\left(\left(K_{(n)}^{*}\right)^{*} \not \subset K_{1}\right)=\mathbb{P}_{\mu_{q}}\left(K^{(n)} \not \subset K_{1}\right) \ll \alpha^{n} .
$$

Hence we have

$$
\begin{aligned}
\mathbb{E}_{\mu_{q}}( & \left.W\left(K^{(n)} \cap K_{1}\right)-W(K)\right) \\
& =\mathbb{E}_{\mu_{q}}\left(\mathbf{1}\left\{K^{(n)} \subset K_{1}\right\}\left(W\left(K^{(n)}\right)-W(K)\right)\right)+O\left(\alpha^{n}\right) \\
& =2 \cdot \mathbb{E}_{\varrho, K^{*}}\left(\mathbf{1}\left\{\left(K_{(n)}^{*}\right)^{*} \subset K_{1}\right\} \int_{K^{*} \backslash K_{(n)}^{*}} \lambda(x) d x\right)+O\left(\alpha^{n}\right) \\
& =2 \cdot \mathbb{E}_{\varrho, K^{*}}\left(\int_{K^{*} \backslash K_{(n)}^{*}} \lambda(x) d x\right)+O\left(\alpha^{n}\right),
\end{aligned}
$$

where we used that $\lambda$ is integrable. Therefore, by Theorem 3.1, we have

$$
\begin{aligned}
\lim _{n \rightarrow \infty} & n^{2 /(d+1)} \mathbb{E}_{\mu_{q}}\left(W\left(K^{(n)} \cap K_{1}\right)-W(K)\right) \\
& =2 \cdot \lim _{n \rightarrow \infty} n^{2 /(d+1)} \mathbb{E}_{\varrho, K^{*}} \int_{K^{*} \backslash K_{(n)}^{*}} \lambda(x) d x \\
& =2 c_{d} \int_{\partial K^{*}} \varrho(x)^{-2 /(d+1)} \lambda(x) \kappa^{*}(x)^{1 /(d+1)} \mathcal{H}^{d-1}(d x) \\
& =2 c_{d} \omega_{d}^{-(d-1) /(d+1)} \int_{\partial K^{*}} \tilde{q}(x)^{-2 /(d+1)}\|x\|^{-d+1} \kappa^{*}(x)^{1 /(d+1)} \mathcal{H}^{d-1}(d x),
\end{aligned}
$$

where $\kappa^{*}$ denotes the generalized Gauss curvature of $K^{*}$. In the following, for $x \in \partial K$, let $\sigma_{K}(x)$ denote an exterior unit normal vector of $K$ at $x$. It is unique for $\mathcal{H}^{d-1}$-almost all $x \in \partial K$.

Theorem 5.2. Let $K \subset \mathbb{R}^{d}$ be a convex body with $o \in \operatorname{int}(K)$ and let $q:[0, \infty) \times S^{d-1} \rightarrow$ $[0, \infty)$ be a measurable function satisfying (q1)-(q3). Then

$$
\begin{aligned}
\lim _{n \rightarrow \infty} & n^{2 /(d+1)} \mathbb{E}_{\mu_{q}}\left(W\left(K^{(n)} \cap K_{1}\right)-W(K)\right) \\
& =2 c_{d} \omega_{d}^{-(d-1) /(d+1)} \int_{\partial K} q\left(h\left(K, \sigma_{K}(x)\right), \sigma_{K}(x)\right)^{-2 /(d+1)} \kappa(x)^{d /(d+1)} \mathcal{H}^{d-1}(d x) .
\end{aligned}
$$

The proof is completed in Section 6 by means of Lemma 6.2 .

Example. Observe that if $q:\left\{(h(K, u), u) \in(0, \infty) \times S^{d-1}: u \in S^{d-1}\right\} \rightarrow[0, \infty)$ is positive and continuous, then $q$ can be extended to $[0, \infty) \times S^{d-1}$ such that (q1)-(q3) are satisfied. For any such extension, the right-hand side of (5.2) remains unchanged. As an example, we may choose $q_{1}$ such that $q_{1}(t, u)=t^{\left(d^{2}-1\right) / 2}$ for $t=h(K, u)$ and $u \in S^{d-1}$. Then the integral in (5.2) turns into

$$
\int_{\partial K} \frac{\kappa(x)^{d /(d+1)}}{\left\langle x, \sigma_{K}(x)\right\rangle^{d-1}} \mathcal{H}^{d-1}(d x)=\Omega_{d^{2}}(K),
$$


where

$$
\Omega_{p}(K):=\int_{\partial K} \frac{\kappa(x)^{p /(d+p)}}{\left\langle x, \sigma_{K}(x)\right\rangle^{(p-1) d /(d+p)}} \mathcal{H}^{d-1}(d x)
$$

is the $p$-affine surface area of $K$ (see $[\mathbf{1 8}, \mathbf{1 9}, \mathbf{2 3 - 2 5}, \mathbf{2 7}, \mathbf{4 7}, \mathbf{4 8}])$. It has been shown that $\Omega_{d^{2}}(K)=\Omega_{1}\left(K^{*}\right)$; see [19]. Moreover, for a convex body $L \subset \mathbb{R}^{d}$, the equiaffine isoperimetric inequality states that

$$
\Omega_{1}(L) \leqslant d \alpha_{d}^{2 /(d+1)} V(L)^{(d-1) /(d+1)}
$$

with equality if and only if $L$ is an ellipsoid (cf. $[\mathbf{7}, \mathbf{1 8}, \mathbf{2 6 - 2 8}])$. Thus we get

$$
\lim _{n \rightarrow \infty} n^{2 /(d+1)} \mathbb{E}_{\mu_{q_{1}}}\left(W\left(K^{(n)} \cap K_{1}\right)-W(K)\right) \leqslant 2 d c_{d} \omega_{d}^{-(d-1) /(d+1)} \alpha_{d}^{2 /(d+1)} V\left(K^{*}\right)^{(d-1)(d+1)}
$$

with equality if and only if $K^{*}$ is an ellipsoid, that is, if and only if $K$ is an ellipsoid. This can be interpreted as saying that, among all convex bodies for which the volume of the polar body is fixed, ellipsoids are worst approximated asymptotically by circumscribed random polytopes (with respect to the density $q_{1}$ ) in the sense of the mean width.

For another application, we define

$$
F(P):=f_{d-1}(P),
$$

for a convex polyhedral set $P \subset \mathbb{R}^{d}$. It is well known that $f_{0}(P)=f_{d-1}\left(P^{*}\right)$ for a convex polytope $P \subset \mathbb{R}^{d}$ with $o \in \operatorname{int}(P)$. Thus, from Proposition 5.1, we get

$$
\begin{aligned}
\mathbb{E}_{\mu_{q}}\left(f_{d-1}\left(K^{(n)}\right)\right)= & \mathbb{E}_{\varrho, K^{*}}\left(f_{d-1}\left(\left(K_{(n)}^{*}\right)^{*}\right)\right) \\
= & \mathbb{E}_{\varrho, K^{*}}\left(\mathbf{1}\left\{\left(K_{(n)}^{*}\right)^{*} \subset K_{1}\right\} f_{d-1}\left(\left(K_{(n)}^{*}\right)^{*}\right)\right) \\
& \quad+\mathbb{E}_{\varrho, K^{*}}\left(\mathbf{1}\left\{\left(K_{(n)}^{*}\right)^{*} \not \subset K_{1}\right\} f_{d-1}\left(\left(K_{(n)}^{*}\right)^{*}\right)\right) \\
= & \mathbb{E}_{\varrho, K^{*}}\left(\mathbf{1}\left\{\left(K_{(n)}^{*}\right)^{*} \subset K_{1}\right\} f_{0}\left(K_{(n)}^{*}\right)\right)+O\left(n \cdot \alpha^{n}\right) \\
= & \mathbb{E}_{\varrho, K^{*}}\left(f_{0}\left(K_{(n)}^{*}\right)\right)+O\left(n \cdot \alpha^{n}\right),
\end{aligned}
$$

where $\alpha \in(0,1)$ is a suitable constant.

The following Theorem 5.3 generalizes Theorem 2.1 in the same way as Theorem 5.2 extends Theorem 2.2 .

Theorem 5.3. Let $K \subset \mathbb{R}^{d}$ be a convex body with $o \in \operatorname{int}(K)$ and let $q:[0, \infty) \times S^{d-1} \rightarrow$ $[0, \infty)$ be a measurable function satisfying (q1)-(q3). Then

$$
\begin{aligned}
& \lim _{n \rightarrow \infty} n^{-(d-1) /(d+1)} \mathbb{E}_{\mu_{q}}\left(f_{d-1}\left(K^{(n)}\right)\right) \\
& \quad=c_{d} \omega_{d}^{-(d-1) /(d+1)} \int_{\partial K} q\left(h\left(K, \sigma_{K}(x)\right), \sigma_{K}(x)\right)^{(d-1) /(d+1)} \kappa(x)^{d /(d+1)} \mathcal{H}^{d-1}(d x) .
\end{aligned}
$$

The proof follows by applying Corollary 3.2 and Lemma 6.2 .

\section{Polarity and an integral transformation}

In this section, we establish the required integral transformation involving the generalized Gauss curvatures of a convex body and its polar body. The main difficulty of the proof is due to the fact that we do not make any smoothness assumptions on the convex bodies that are considered. 
Let $L \subset \mathbb{R}^{d}$ be a convex body. If the support function $h_{L}$ of $L$ is differentiable at $u \neq o$, then the gradient $\nabla h_{L}(u)$ of $h_{L}$ at $u$ is equal to the unique boundary point of $L$ having $u$ as an exterior normal vector. In particular, the gradient of $h_{L}$ is a function that is homogeneous of degree zero. Note that $h_{L}$ is differentiable at $\mathcal{H}^{d-1}$-almost all unit vectors. We write $D_{d-1} h_{L}(u)$ for the product of the principal radii of curvature of $L$ in direction $u \in S^{d-1}$, whenever the support function $h_{L}$ is twice differentiable in the generalized sense at $u \in S^{d-1}$. Note that this is the case for $\mathcal{H}^{d-1}$-almost all $u \in S^{d-1}$. The Gauss map $\sigma_{L}$ is defined $\mathcal{H}^{d-1}$-almost everywhere on $\partial L$. If $\sigma_{L}$ is differentiable in the generalized sense at $x \in \partial L$, which is the case for $\mathcal{H}^{d-1}$ almost all $x \in \partial L$, then the product of the eigenvalues of the differential is the Gauss curvature $\kappa_{L}(x)$. The connection to curvatures defined on the generalized normal bundle $\mathcal{N}(L)$ of $L$ will be used in the following proof (cf. [20]).

Lemma 6.1. Let $L \subset \mathbb{R}^{d}$ be a convex body containing the origin in its interior. If $g: \partial L \rightarrow$ $[0, \infty]$ is measurable, then

$$
\int_{\partial L} g(x) \kappa_{L}(x)^{1 /(d+1)} \mathcal{H}^{d-1}(d x)=\int_{S^{d-1}} g\left(\nabla h_{L}(u)\right) D_{d-1} h_{L}(u)^{d /(d+1)} \mathcal{H}^{d-1}(d u) .
$$

Proof. In the following proof, we use results and methods from [20], to which we refer the reader for additional references and detailed definitions. Let $\mathcal{N}(L)$ denote the generalized normal bundle of $L$ and let $k_{i}(x, u) \in[0, \infty]$, with $i=1, \ldots, d-1$, be the generalized curvatures of $L$, which are defined for $\mathcal{H}^{d-1}$-almost all $(x, u) \in \mathcal{N}(L)$. Expressions such as

$$
\frac{k_{i}(x, u)^{1 /(d+1)}}{\sqrt{1+k_{i}(x, u)^{2}}} \quad \text { or } \quad \frac{k_{i}(x, u)}{\sqrt{1+k_{i}(x, u)^{2}}}
$$

with $k_{i}(x, u)=\infty$ are understood as limits as $k_{i}(x, u) \rightarrow \infty$, and yield 0 or 1 , respectively, in the two given examples. As is common in measure theory, the product $0 \cdot \infty$ is defined as 0 .

Our starting point is the expression

$$
I:=\int_{\mathcal{N}(L)} g(x) \prod_{i=1}^{d-1} \frac{k_{i}(x, u)^{1 /(d+1)}}{\sqrt{1+k_{i}(x, u)^{2}}} \mathcal{H}^{d-1}(d(x, u)),
$$

which will be evaluated in two different ways. A comparison of the resulting expressions yields the assertion of the lemma.

First, we rewrite $I$ in the form

$$
I=\int_{\mathcal{N}(L)} g(x)\left(\prod_{i=1}^{d-1} k_{i}(x, u)\right)^{-d /(d+1)} J_{d-1} \pi_{2}(x, u) \mathcal{H}^{d-1}(d(x, u)),
$$

where

$$
J_{d-1} \pi_{2}(x, u)=\prod_{i=1}^{d-1} \frac{k_{i}(x, u)}{\sqrt{1+k_{i}(x, u)^{2}}}
$$

for $\mathcal{H}^{d-1}$-almost all $(x, u) \in \mathcal{N}(L)$, is the (approximate) Jacobian of the map $\pi_{2}: \mathcal{N}(L) \rightarrow$ $S^{d-1},(x, u) \mapsto u$. To check $(6.2)$, we distinguish the following cases. If $k_{i}(x, u)=0$ for some $i$, then the integrands on the right-hand sides of (6.1) and (6.2) are zero, since $0 \cdot \infty=0$ and $J_{d-1} \pi_{2}(x, u)=0$. If $k_{i}(x, u) \neq 0$ for all $i$ and $k_{j}(x, u)=\infty$ for some $j$, then again both integrands are zero. In all other cases the assertion is clear. 
For $\mathcal{H}^{d-1}$-almost all $u \in S^{d-1}$, we see that $\nabla h_{L}(u) \in \partial L$ is the unique boundary point of $L$ which has $u$ as an exterior unit normal vector. Then the coarea formula yields

$$
I=\int_{S^{d-1}} g\left(\nabla h_{L}(u)\right)\left(\prod_{i=1}^{d-1} k_{i}\left(\nabla h_{L}(u), u\right)\right)^{-d /(d+1)} \mathcal{H}^{d-1}(d u) .
$$

Using [20, Lemma 3.4], we get

$$
I=\int_{S^{d-1}} g\left(\nabla h_{L}(u)\right) D_{d-1} h_{L}(u)^{d /(d+1)} \mathcal{H}^{d-1}(d u) .
$$

Now we consider also the projection $\pi_{1}: \mathcal{N}(L) \rightarrow \partial L,(x, u) \mapsto x$, which has the (approximate) Jacobian

$$
J_{d-1} \pi_{1}(x, u)=\prod_{i=1}^{d-1} \frac{1}{\sqrt{1+k_{i}(x, u)^{2}}},
$$

for $\mathcal{H}^{d-1}$-almost all $(x, u) \in \mathcal{N}(L)$. A similar argument as before yields

$$
\begin{aligned}
I & =\int_{\mathcal{N}(L)} g(x)\left(\prod_{i=1}^{d-1} k_{i}(x, u)\right)^{1 /(d+1)} J_{d-1} \pi_{1}(x, u) \mathcal{H}^{d-1}(d(x, u)) \\
& =\int_{\partial L} g(x)\left(\prod_{i=1}^{d-1} k_{i}\left(x, \sigma_{L}(x)\right)\right)^{1 /(d+1)} \mathcal{H}^{d-1}(d x) .
\end{aligned}
$$

By [20, Lemma 3.1], we also get

$$
I=\int_{\partial L} g(x) \kappa_{L}(x)^{1 /(d+1)} \mathcal{H}^{d-1}(d x) .
$$

A comparison of equations (6.3) and (6.4) gives the required equality.

REMARK. An alternative argument can be based on arguments similar to those used in [18] for the proof of the equality of two representations of the affine surface area of a convex body.

Lemma 6.2. Let $K \subset \mathbb{R}^{d}$ be a convex body with $o \in \operatorname{int}(K)$. If $f:[0, \infty) \times S^{d-1} \rightarrow[0, \infty)$ is a measurable function and $\tilde{f}(x):=f\left(\|x\|^{-1},\|x\|^{-1} x\right)$, with $x \in \partial K^{*}$, then

$$
\int_{\partial K^{*}} \tilde{f}(x)\|x\|^{-d+1} \kappa^{*}(x)^{1 /(d+1)} \mathcal{H}^{d-1}(d x)=\int_{\partial K} f\left(h\left(K, \sigma_{K}(x)\right), \sigma_{K}(x)\right) \kappa(x)^{d /(d+1)} \mathcal{H}^{d-1}(d x) .
$$

Proof. We apply Lemma 6.1 with $L=K^{*}$ and $g(x)=\tilde{f}(x)\|x\|^{-d+1}$, with $x \in \partial K^{*}$, and thus we get

$$
\begin{aligned}
\int_{\partial K^{*}} & \tilde{f}(x)\|x\|^{-d+1} \kappa^{*}(x)^{1 /(d+1)} \mathcal{H}^{d-1}(d x) \\
\quad= & \int_{S^{d-1}} \tilde{f}\left(\nabla h_{K^{*}}(u)\right)\left\|\nabla h_{K^{*}}(u)\right\|^{-d+1} D_{d-1} h_{K^{*}}(u)^{d /(d+1)} \mathcal{H}^{d-1}(d u) .
\end{aligned}
$$

Next we apply Theorem 2.2 in [19] (or the second part of [21, Corollary 5.1]). Thus, using the fact that, for $\mathcal{H}^{d-1}$-almost all $u \in S^{d-1}$, we have that $h_{K^{*}}$ is differentiable in the generalized 
sense at $u$ and $\rho(K, u) u$ is a normal boundary point of $K$, we have:

$$
D_{d-1} h_{K^{*}}(u)^{d /(d+1)}=\kappa(x)^{d /(d+1)}\left\langle u, \sigma_{K}(x)\right\rangle^{-d},
$$

where $x=\rho(K, u) u \in \partial K$ and $u=\|x\|^{-1} x \in S^{d-1}$. Hence, we have

$$
\begin{aligned}
& \int_{\partial K^{*}} \tilde{f}(x)\|x\|^{-d+1} \kappa^{*}(x)^{1 /(d+1)} \mathcal{H}^{d-1}(d x) \\
& \quad=\int_{S^{d-1}} \tilde{f}\left(\nabla h_{K^{*}}(u)\right) \frac{\left\|\nabla h_{K^{*}}(u)\right\|^{-d+1}}{\left\langle u, \sigma_{K}(\rho(K, u) u)\right\rangle^{d}} \kappa(\rho(K, u) u)^{d /(d+1)} \mathcal{H}^{d-1}(d x) .
\end{aligned}
$$

The bijective and bilipschitz transformation $T: S^{d-1} \rightarrow \partial K, u \mapsto \rho(K, u) u$, has the Jacobian

$$
J T(u)=\frac{\left\|\nabla h_{K^{*}}(u)\right\|}{h_{K^{*}}(u)^{d}}
$$

for $\mathcal{H}^{d-1}$-almost all $u \in S^{d-1}$ (see the proof of [19, Lemma 2.4]). Therefore, we have

$$
\begin{aligned}
\int_{\partial K^{*}} & \tilde{f}(x)\|x\|^{-d+1} \kappa^{*}(x)^{1 /(d+1)} \mathcal{H}^{d-1}(d x) \\
& =\int_{\partial K} \tilde{f}\left(\nabla h_{K^{*}}\left(\frac{x}{\|x\|}\right)\right) \frac{\left\|\nabla h_{K^{*}}(x /\|x\|)\right\|^{-d}}{\left\langle x /\|x\|, \sigma_{K}(x)\right\rangle^{d}} h_{K^{*}}\left(\frac{x}{\|x\|}\right)^{d} \kappa(x)^{d /(d+1)} \mathcal{H}^{d-1}(d x) \\
& =\int_{\partial K} \tilde{f}\left(\nabla h_{K^{*}}(x)\right) \frac{\left\|\nabla h_{K^{*}}(x)\right\|^{-d}}{\left\langle x, \sigma_{K}(x)\right\rangle^{d}} h_{K^{*}}(x)^{d} \kappa(x)^{d / d+1} \mathcal{H}^{d-1}(d x) \\
& =\int_{\partial K} f\left(\left\|\nabla h_{K^{*}}(x)\right\|^{-1}, \nabla h_{K^{*}}(x) /\left\|\nabla h_{K^{*}}(x)\right\|\right) \kappa(x)^{d /(d+1)} \mathcal{H}^{d-1}(d x), \\
& =\int_{\partial K} f\left(h_{K}\left(\sigma_{K}(x)\right), \sigma_{K}(x)\right) \kappa(x)^{d /(d+1)} \mathcal{H}^{d-1}(d x),
\end{aligned}
$$

since $h_{K^{*}}(x)=1$ for $x \in \partial K$ and $x^{*}:=\nabla h_{K^{*}}(x)$ satisfies $\left\|x^{*}\right\|^{-1}=\left\langle x, \sigma_{K}(x)\right\rangle$ as well as $x^{*} /\left\|x^{*}\right\|=\sigma_{K}(x)$, for $\mathcal{H}^{d-1}$-almost all $x \in \partial K$.

Note. In Theorem 3.1 and Corollary 3.2 the assumptions on $\lambda$ and $\varrho$ can be slightly weakened. It is sufficient to assume that the integrable function $\lambda$ and the probability density $\varrho$ are continuous and $\varrho$ is positive, at each boundary point of $K$. This follows from a compactness argument that shows that $\lambda$ is bounded and $\varrho$ is bounded from above and from below by a positive constant in a suitable neighborhood of $\partial K$. A compactness argument also yields the continuity properties of $\lambda$ and $\varrho$ that are actually needed in the proofs. A similar remark applies to property (q2) in Section 5.

Acknowledgements. We are grateful for stimulating discussions with Rolf Schneider. The third author is grateful to Joseph Yukich for having pointed out to him that the weaker continuity conditions that are discussed in the preceding Note are possible in the context of mean and variance asymptotics for the vertex count of a sample in the unit ball; see, for example, [39, Theorem 2.1].

\section{References}

1. I. BÁRÁNY, 'Random polytopes in smooth convex bodies', Mathematika 39 (1992) 81-92.

2. I. BÁRÁNY and CH. BuCHTA, 'Random polytopes in a convex polytope, independence of shape, and concentration of vertices', Math. Ann. 297 (1993) 467-497. 
3. I. Barany and M. Reitzner, 'On the variance of random polytopes', Adv. Math., to appear, http://www.renyi.hu/ barany/cikkek/var-aim.pdf.

4. I. Barany and M. Reitzner, 'Poisson polytopes', Ann. Probab., to appear, http://www.renyi.hu/ $\sim$ barany/cikkek/poi-pol2.pdf.

5. I. BÁrÁny and V. Vu, 'Central limit theorems for Gaussian polytopes', Ann. Probab. 35 (2007) 1593-1621.

6. K. H. Borgwardt, The simplex method, a probabilistic analysis (Springer, Berlin, 1987).

7. K. Böröczky JR., 'Stability of the Blaschke-Santaló and the affine isoperimetric inequality', Adv. Math., to appear.

8. K. Böröczky JR. and M. Reitzner, 'Approximation of smooth convex bodies by random circumscribed polytopes', Ann. Appl. Probab. 14 (2004) 239-273.

9. K. Böröczky JR. and R. Schneider, 'The mean width of circumscribed random polytopes', Canadian Math. Bull., to appear.

10. K. J. BöröCzky, F. Fodor, M. Reitzner and V. VíGH, 'Mean width of random polytopes in a reasonably smooth convex body', J. Multivariate Anal. 100 (2009) 2287-2295.

11. K. J. Böröczky, L.M. Hoffmann and D. Hug, 'Expectation of intrinsic volumes of random polytopes', Period. Math. Hungar. 57 (2008) 143-164.

12. L. DümbGen and G. Walther, 'Rates of convergence for random approximations of convex sets', Adv. in Appl. Probab. 28 (1996) 384-393.

13. B. Efron, 'The convex hull of a random set of points', Biometrika 52 (1965) 331-343.

14. H. Federer, Geometric measure theory (Springer, Berlin, 1969).

15. S. Glasauer and P. M. Gruber, 'Asymptotic estimates for best and stepwise approximation of convex bodies III', Forum Math. 9 (1997) 383-404.

16. P. M. Gruber, 'Comparisons of best and random approximations of convex bodies by polytopes', Rend. Circ. Mat. Palermo (2) Suppl. 50 (1997) 189-216.

17. P. M. Gruber, Convex and discrete geometry (Springer, Berlin, 2007).

18. D. Hug, 'Contributions to affine surface area', Manuscripta Math. 91 (1996) 283-301.

19. D. Hug, 'Curvature relations and affine surface area for a general convex body and it polar', Results Math. 29 (1996) 233-248.

20. D. Hug, 'Absolute continuity for curvature measures of convex sets I', Math. Nachr. 195 (1998) 139-158.

21. D. Hug, 'Absolute continuity for curvature measures of convex sets III', Adv. Math. 169 (2002) 92-117.

22. F.J. Kaltenbach, 'Asymptotisches Verhalten zufälliger konvexer Polyeder', Doctoral Thesis, AlbertLudwigs-Universität, Freiburg i. Br., 1990.

23. K. Leichtweiss, Affine geometry of convex bodies (Johann Ambrosius Barth Verlag, Heidelberg, 1998).

24. M. Ludwig and M. Reitzner, 'A characterization of affine surface area', Adv. Math. 147 (1999) 138-172.

25. M. Ludwig and M. Reitzner, 'A classification of $\operatorname{SL}(n)$ invariant valuations', Ann. of Math. to appear, http://pjm.math.berkeley.edu/annals/ta/090218-Reitzner/090218-Reitzner-v2.pdf.

26. E. LUTWAK, 'Selected affine isoperimetric inequalities', Handbook of convex geometry (eds P. M. Gruber and J. M. Wills; North-Holland, Amsterdam, 1993), 151-176.

27. E. Lutwak, 'The Brunn-Minkowski-Firey theory. II. Affine and geominimal surface areas', Adv. Math. 118 (1996) 244-294.

28. C. M. PetTy, 'Affine isoperimetric problems', Discrete geometry and convexity (eds J. E. Goodman, E. Lutwak, J. Malkevitch and R. Pollack), Annals of New York Academy of Sciences 440 (New York Academy of Sciences, New York, 1985), 113-127.

29. M. Reitzner, 'Random polytopes and the Efron-Stein jackknife inequality', Ann. Probab. 31 (2003) 21362166 .

30. M. Reitzner, 'Central limit theorems for random polytopes', Probab. Theory Related Fields 133 (2005) $483-507$.

31. A. RÉnyi and R. Sulanke, 'Über die konvexe Hülle von $n$ zufällig gewählten Punkten', Z. Wahrscheinlichkeitsth. verw. Geb. 2 (1963) 75-84.

32. A. RÉnyi and R. Sulanke, 'Über die konvexe Hülle von $n$ zufällig gewählten Punkten. II', $Z$. Wahrscheinlichkeitsth. verw. Geb. 3 (1964) 138-147.

33. A. RÉnyi and R. Sulanke, 'Zufällige konvexe Polygone in einem Ringgebiet', Z. Wahrscheinlichkeitsth. verw. Geb. 9 (1968) 146-157.

34. R. Schneider, 'Random approximation of convex sets', J. Microsc. 151 (1988) 211-227.

35. R. Schneider, Convex bodies: the Brunn-Minkowski theory (Cambridge University Press, Cambridge, 1993).

36. R. Schneider, 'Discrete aspects of stochastic geometry', Handbook of discrete and computational geometry, 2nd edn (eds. J. E. Goodman and J. O'Rourke; CRC Press, Boca Raton, 2004), 255-278.

37. R. Schneider and W. Weil, Stochastic and integral geometry, Probability and its Applications (Springer, Berlin, 2008).

38. R. Schneider and J. A. Wieacker, 'Random polytopes in a convex body', Z. Wahrscheinlichkeitsth. verw. Geb. 52 (1980) 69-73.

39. T. Schreiber and J. Yukich, 'Variance asymptotics and central limit theorems for generalized growth processes with applications to convex hulls and maximal points', Ann. Probab. 36 (2008) 363-396.

40. C. Schütт, 'Random polytopes and affine surface area', Math. Nachr. 170 (1994) 227-249.

41. C. Schütt and E. Werner, 'The convex floating body', Math. Scand. 66 (1990) 275-290.

42. C. Schütt and E. Werner, 'Polytopes with vertices chosen randomly from the boundary of a convex 
body', Geometric aspects of functional analysis, Lecture Notes in Mathematics 1807 (Springer, Berlin, 2003) 241-422

43. J. J. Sylvester, 'Problem 1491', Educational Times (London), April 1864 1-28.

44. V. VU, 'Sharp concentration of random polytopes', Geom. Funct. Anal. 15 (2005) 1284-1318.

45. V. VU, 'Central limit theorems for random polytopes in a smooth convex set', Adv. Math. 207 (2006) 221-243.

46. W. Weil and J. A. Wieacker, 'Stochastic geometry', Handbook of convex geometry (eds. P. M. Gruber and J. M. Wills; North-Holland, Amsterdam 1993), 1391-1438.

47. E. Werner, 'On $L_{p}$ affine surface areas', Indiana Univ. Math. J. 56 (2007) 2305-2324.

48. E. Werner and D. Ye, 'New $L_{p}$ affine isoperimetric inequalities', Adv. Math. 218 (2008) 762-780.

49. J. A. Wieacker, 'Einige Probleme der polyedrischen Approximation', Diplomarbeit, Albert-LudwigsUniversität, Freiburg i. Br., 1978.

50. H. Ziezold, 'Über die Eckenanzahl zufälliger konvexer Polygone', Izv. Akad. Nauk Armjan. SSR Ser. Mat. 5 (1970) 296-312.

Károly J. Böröczky

Alfréd Rényi Institute of Mathematics

Hungarian Academy of Sciences

PO Box 127

H-1364 Budapest

Hungary

and

Department of Geometry

Roland Eötvös University

Pázmány Péter sétány 1/C

H-1117 Budapest

Hungary

carlos@renyi.hu

Daniel Hug

Karlsruhe Institute of Technology

Department of Mathematics

D-76128 Karlsruhe

Germany

daniel.hug@kit.edu
Ferenc Fodor

Department of Geometry

University of Szeged

Aradi vértanúk tere 1

H-6720 Szeged

Hungary

and

Department of Mathematics and Statistics University of Calgary

2500 University Dr. N.W.

Calgary, Alberta

Canada T2N 1N4

fodorf@math.u-szeged.hu

fodor@math.ucalgary.ca 